\title{
Fluid Gauge Theory
}

By Tsutomu Kambe

University of Tokyo

Abstract- According to the general gauge principle, Fluid Gauge Theory is presented to cover a wider class of flow fields of a perfect fluid without internal energy dissipation under anisotropic stress field. Thus, the theory of fluid mechanics is extended to cover time dependent rotational flows under anisotropic stress field of a compressible perfect fluid, including turbulent flows. Eulerian fluid mechanics is characterized with isotropic pressure stress fields. The study is motivated from three observations. First one is experimental observations reporting large-scale structures coexisting with turbulent flow fields. This encourages a study of how such structures observed experimentally are possible in turbulent shear flows, Second one is a theoretical and mathematical observation: the "General solution to Euler's equation of motion" (found by Kambe in 2013) predicts a new set of four background-fields, existing in the linked $4 d$-spacetime. Third one is a physical query, "what symmetry implies the current conservation law ?". The latter two observations encourage a gauge-theoretic formulation by defining a differential one-form representing the interaction between the fluid-current field $j \mu$ and a background field $a \mu$.

Keywords: anisotropic stress field, gauge principle, fluid gauge theory, current conservation, euler equation.

GJSFR-A Classification: FOR Code: 020399

Strictly as per the compliance and regulations of:

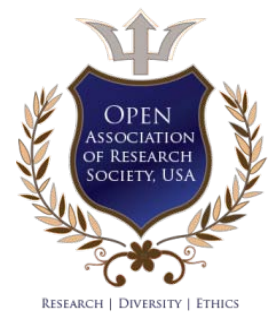

(C) 2021. Tsutomu Kambe. This research/review article is distributed under the terms of the Attribution-NonCommercialNoDerivatives 4.0 International (CC BY-NC-ND 4.0). You must give appropriate credit to authors and reference this article if parts of the article are reproduced in any manner. Applicable licensing terms are at https://creativecommons.org/ licenses/by-nc$\mathrm{nd} / 4.0 \%$ 


\title{
Fluid Gauge Theory
}

\author{
Tsutomu Kambe
}

\begin{abstract}
According to the general gauge principle, Fluid Gauge Theory is presented to cover a wider class of flow fields of a perfect fluid without internal energy dissipation under anisotropic stress field. Thus, the theory of fluid mechanics is extended to cover time dependent rotational flows under anisotropic stress field of a compressible perfect fluid, including turbulent flows. Eulerian fluid mechanics is characterized with isotropic pressure stress fields. The study is motivated from three observations. First one is experimental observations reporting largescale structures coexisting with turbulent flow fields. This encourages a study of how such structures observed experimentally are possible in turbulent shear flows, Second one is a theoretical and mathematical observation: the "General solution to Euler's equation of motion" (found by Kambe in 2013) predicts a new set of four background-fields, existing in the linked $4 d$-spacetime. Third one is a physical query, "what symmetry implies the current conservation law ?". The latter two observations encourage a gauge-theoretic formulation by defining a differential one-form representing the interaction between the fluid-current field $j^{\mu}$ and a background field $a_{\mu}$. A known relativistic action of a perfect fluid is introduced together with the interaction action just mentioned, and furthermore, a third gauge invariant action is defined to govern the field $a_{\mu}$ linearly in its free-state. The general gauge principle is applied to the combined system of the three actions to describe general time-dependent rotational flow fields of an ideal compressible fluid. The combined system can be shown to be invariant under both global and local gauge transformations of variations of $a_{\mu}$. The global gauge transformation is a diagnostic test whether the system is receptive to a new field $a_{\mu}\left(x^{\nu}\right)$. Since the test is cleared, a new internal stress field $M_{i k}\left(x^{\nu}\right)$ is introduced into the flow field of a perfect fluid, together with the current conservation $\partial_{\mu} j^{\mu}=0$, where the stress $M_{i k}$ is an anisotropic stress field which is an extension added to the Eulerian isotropic pressure-stress field $p \delta_{i k}$.

Keywords: anisotropic stress field, gauge principle, fluid gauge theory, current conservation, Euler equation.
\end{abstract}

\section{INTRODUCTION}

\section{a) Background of present research}

Gauge invariance is one of the fundamental symmetries in modern theoretical physics. It took almost a century for transition from the 19th-century recognition of a mathematical invariance existing in classical electromagnetic theory to the 20thcentury recognition of its fundamental physical significance. Real recognition of the gauge symmetry and its physical significance required two new fields developed in the 20th century: the relativity theory for physics of the world structure of linked 4dspacetime and the quantum mechanics for the new dimension of a phase factor in complex representation of wave function. The 20th-century recognition resulted in the naming of the invariance as gauge invariance and in successful formulation of the Gauge Principle. Its historical development is reviewed by Kambe (2021a) concerning its gradual and zigzag developmental processes in quantum electrodynamics (QED). The gauge theory played vital roles in modern particle physics which was revolutionary (e.g. Aitchison \& Hey (2013), Utiyama (1956)). The same paper (Kambe 2021a) presents also reviews of the gauge invariances existing in the two theories of the weak gravitational field and the electromagnetic theory with emphasizing the similarity between them. In addition, its last section 5 presents "Motivations for Fluid Gauge Theory". Thus, on the basis of these backgrounds of gauge theories reviewed in the article (Kambe 2021a), the present paper proposes possible application of the gauge theory to fluid flows although the field of fluid-flow is not listed in the literatures reviewed.

Author: (Former Professor at) Department of Physics, University of Tokyo, Tokyo, Japan, (Home) Higashi-yama 2-11-3, Meguroku, Tokyo, Japan. e-mail: kambe@ruby.dti.ne.jp 
From the aspects of fluid flows, recent studies of wall turbulence and shear flow turbulence recognize existence of large scale structures. It is known from experimental studies that periodic waves exist robustly in the background irregularly fluctuating flow field, and streak structures are observed to coexist with turbulent field. The streaks in turbulence are wavy streamwise vortices surrounded by a sea of incoherent turbulent motions. The observed large scale structures are characterized by streamwise streaks and long meandering vortical structures. Concerning these experimental studies, see Reynolds \& Hussain (1972); Kim \& Adrian (1999); Del Álamo \& Jiménez (2006); Monty et al. (2007); Hutchins \& Marusic (2007); Smits et al. (2011).

Related mathematical aspect was investigated by Scofield and Huq (2014), stating that the conservation law of current flux implies existence of a wavy field governed by Maxwell-type equations, proposing that this might be applied to transverse travelling waves observed in turbulent internal flows along a spiral pipe. The theory is based on four conservation equations of energy and momentum of the whole system.

In order to highlight such aspects of large-scale structures coexisting with irregularly fluctuating flow field and contemplate how such structures observed experimentally are possible in turbulent shear flows, Kambe (2017) proposed a new scenario of turbulence theory, based on the view that the entire physical system is composed of two fields: fluid-flow field and transverse-wave field. General formalism of theoretical physics is applied to the study of whole system consisting of a flow field and a wave field, with two Lagrangian densities corresponding to each component and additional Lagrangian expressing their interaction. This approach yielded good results that are consistent with observations.

In particular, it is remarked that the "fluid gauge theory to be presented here supports the above theoretical approach proposed by Kambe (2017). In addition, the most recent research of Kambe (2020) sheds light on physical mechanism how and why the entire physical system has a structure composed of two fields: a flow field and a wavy gauge field. The underlying key is the inseparable relation between the mass conservation law and a gauge symmetry. Namely, the current conservation must be satisfied at every point and every time by the flow field, which is to be ensured by a background gauge field. This is the physical idea requiring the system to be composed of two fields of a flow and a background gauge field.

The new system consisting of a fluid-flow and a background field ensuring current conservation is powerful. It is likely that this enables to resolve an issue historically unresolved, that is the problem of "Dust striations observed in the resonance-tube experiment" by August Kundt (1866), where there exists two different length scales with their ratio more than fifty. The larger one correponds to the wavelength of the resonant acoustic wave. A recent numerical test study based on the present new system gives encouraging outputs on the smaller scales (see the section $\S 4$ Summary and discussions).

As investigated in Kambe (2020), according to the current formulation of fluid mechanics, from a single relativistic energy equation of fluid motion, two conservation equations are obtained in the non-relativistic limit : one is the mass conservation and the other is the traditional form of energy equation. This is a riddle (see below at the part c) from the physics point of view. We are particularly concerned with the mass conservation equation and investigate what symmetry implies the mass conservation. A key to resolve this Riddle is provided by the general representation of rotational flows of an ideal compressible fluid satisfying the Euler's equation (the part d), derived by Kambe (2013). This gives us a hint of existence of a set of gauge fields, giving rise to anisotropic stress fields within the flows which are time-dependent and rotational flow fields.

In the present study, the Euler's equation of motion is still valid, but it is remarked that the equation is characterized intrinsically by the pressure stress which is represented 
by isotropic stress fields. The present study of Fluid Gauge Theory predicts that the perfect fluid (i.e. a fluid without dissipative internal mechanism) can accommodate anisotropic stress field as well which can exist in unsteady shear flow fields.

This suggests that our physical system should be a combined system consisting of a fluid flow field and a set of background gauge fields. This aspect and its significance have been already investigated by Kambe $(2017,2020)$. The gauge symmetry of the new background gauge fields ensures the law of mass conservation.

\section{b) Section composition of the paper and mathematical structure of the theory}

In the present paper, Fluid Gauge Theory is presented for a perfect fluid according to the general gauge principle. Section II is a preparative section collecting necessary articles for the theory with the section title: An approach aiming at a fluid gauge theory. Section III presents the main theory with the title, Fluid Gauge Theory and adds a remark section supplementing insufficient parts of the presentation.

On a mathematical point of view, more must be commented on the present approach of the Fluid Gauge Theory. When new fields are taken into consideration, those should be implemented (or absorbed) into the structure of covariant derivatives as connection terms like the terms of Christoffel symbol in the gravity theory or the gauge potentials in the electromagnetic theory. The concept of connection in the mathematics is an essential ingredient of the physical gauge theory. It is a challenging work to implement connection terms in the structure of fluid gauge theory. This is left to the Appendices $\mathrm{B}$ and $\mathrm{C}$, because sufficient mathematical expressions and concepts must be presented to arrive at the goal.

Another aspect of the present system of a perfect fluid must be noted. Our system is free from external forcing and in addition free from any internal mechanism of energy dissipation. From mechanical point of view, free fluid motions are not always described by straight trajectories of time evolution of the mechanical system as a whole, namely their geodesics describing the whole system are curved in general.

In fact, we will see in Appendix C.2 that the free motion of a perfect fluid under a background field $a_{\nu}$ can be described by a geodesic equation representing a curved free dynamics. This is derived by the variational principle that makes the action integrals invariant. Namely the new field of the fluid gauge theory has been taken into the structure of covariant derivatives as connection terms and the free dynamics of a perfect fluid under a background field $a_{\nu}$ is described by the geodesic equation.

These mathematical concepts would make the structure of theory complicated easily. In order to make the storyline of the theory clear and as much as simple, those complicating mathematical factors are left to Appendices B and C. However, the mathematical concepts such as geodesic, covariant derivative, connection, etc. . are absolutely necessary for the theory of Fluid Gauge Theory. It is the reason why the appendices to the present paper get massive.

\section{c) By what symmetry the mass conservation law is implied?}

It is well-known that the energy conservation is associated with the symmetry of time translation of mechanical systems. One of the motivations for proposing a fluid gauge theory is stated by the following question: "What physical symmetry implies the mass conservation law ?" This query is raised in regard to the relativistic equation of energy conservation of fluid flows when its non-relativistic limit is taken (Kambe 2020). In the ordinary fluid-mechanics valid in non-relativistic limit, the mass conservation law is given as valid a priori. However, in the fluid-mechanics of relativity theory, fluid motions are governed by four relativistic conservation equations of energy and 
momentum $\partial_{\nu} T^{\mu \nu}=0$, where $T^{\mu \nu}$ is the stress-energy tensor for $\mu, \nu=0,1,2,3$, $\partial_{\nu} \equiv \partial / \partial x^{\nu}$ and $x^{\nu}=\left(x^{0}, x^{k}\right)$ with $x^{0} \equiv c t$ for $t$ the time. (see [Kambe (2020) $\S 2.2$, 2.3] or [Landau \& Lifshitz, 1987, §133] ). Its space components for $\mu=1,2,3$ represent momentum conservation of three components.

On the other hand, its time component $\partial_{\nu} T^{0 \nu}=0$ represents an energy conservation equation. In the non-relativistic limit as a representative flow velocity $v$ is much less than the light velocity $c(\beta \equiv v / c \rightarrow 0)$, the equation for a perfect fluid of mass density $\rho$ and specific internal energy $\epsilon \ddagger$ can be written in the following form:

$$
\begin{gathered}
0=c^{-1} \partial_{t} \bar{T}^{00}+\partial_{k} \bar{T}^{0 k}=c\left(\partial_{t} \rho+\partial_{k}\left(\rho v^{k}\right)\right)+\frac{1}{c}\left(\partial_{t}(\rho \hat{E})+\partial_{k}\left(\rho v^{k} \hat{H}\right)\right)+O\left(\beta^{3}\right), \\
\hat{E}=\frac{1}{2} v^{2}+\epsilon, \quad \hat{H}=\frac{1}{2} v^{2}+h .
\end{gathered}
$$

where $v^{k}$ is the $k$-th component of fluid velocity for $k=1,2,3$. In the non-relativistic limit as $\beta \rightarrow 0$, we obtain the mass conservation equation from the first term:

$$
\partial_{t} \rho+\partial_{k}\left(\rho v^{k}\right)=0
$$

Then, deleting it, the remaining expression reduces to the energy equation of ordinary fluid mechanics in the limit as $\beta \rightarrow 0$. Thus, we obtain the energy conservation equation of fluid flow (Landau \& Lifshitz (1987), Eq.(6.1)):

$$
\partial_{t}(\rho \hat{E})+\partial_{k}\left(\rho v^{k} \hat{H}\right)=0
$$

Here we have obtained two conservation equations from the single energy equation $\partial_{\nu} T^{0 \nu}=0$. But, the Noether's theorem (Noether 1918) of theoretical physics states 'A symmetry implies a conservation law'. This is a riddle. We must ask a question whether the above is satisfactory. In this paper, we try to propose a resolution to this query.

d) A hint to resolve the riddle: General solution of Euler's equation with helicity

A hint to resolve the Riddle mentioned above is found in the general representation of rotational flows given by Kambe (2013) for an ideal compressible flow solution satisfying the Euler's equation. This solution was derived from the action principle for the action $S^{(\text {Eul-rot) }}$ of non-relativistic flow fields:

$$
\begin{gathered}
S^{(\text {Eul-rot })}=S^{(\mathrm{nR})}+S^{(\mathrm{g}-\mathrm{inv})}=\int \rho \mathrm{d} \mathcal{V}\left[\int \Lambda_{\mathrm{nR}} \mathrm{d} t+\int \Lambda_{\mathrm{Gi}} \mathrm{d} t\right] \\
\Lambda_{\mathrm{nR}}=\frac{1}{2} v^{2}-\epsilon, \quad \Lambda_{\mathrm{Gi}}=-\mathrm{D}_{t} \psi-\mathrm{D}_{t}\langle\boldsymbol{U}, \boldsymbol{Z}\rangle \\
\nabla \cdot(\rho \boldsymbol{Z})=0, \quad \nabla \cdot \boldsymbol{U}=0, \quad \mathrm{D}_{t} \equiv \partial_{t}+\boldsymbol{v} \cdot \boldsymbol{\nabla} \\
\mathcal{L}[\boldsymbol{Z}] \equiv \partial_{t} \boldsymbol{Z}+(\boldsymbol{v} \cdot \nabla) \boldsymbol{Z}-(\boldsymbol{Z} \cdot \nabla) \boldsymbol{v}=0
\end{gathered}
$$

where $\boldsymbol{v}=\left(v^{k}\right)$ is the 3 -velocity vector, $\psi$ a scalar function to be determined, and $\Lambda_{\mathrm{nR}}$ is nothing but the ordinary non-relativistic Lagrangian density, while $\Lambda_{\mathrm{Gi}}$ is a gaugeinvariant Lagrangian newly introduced in the study (Kambe, 2013). Regarding the two 3 -vectors $\boldsymbol{Z}$ and $\boldsymbol{U}$, see the paragraph below. Actually, this study had double aims. One was an attempt to obtain general representation of rotational flow with nonzero helicity (Kambe 2011). Second aim was more fundamental, striving to establish

$\ddagger$ There is no energy dissipation in the perfect fluid, hence no entropy change. Assuming uniform entropy throughout, the internal energy $\epsilon$ depends only on $\rho$. Hence $\epsilon=\epsilon(\rho)$, and $h \equiv \epsilon(\rho)+p / \rho$. 
equivalence between two formulations of Eulerian specification of field variables and the Lagrangian specification under the action principle. Each term of the Lagrangians $\Lambda_{\mathrm{nR}}$ and $\Lambda_{\mathrm{Gi}}$ satisfies local gauge invariance with respect to translation and rotation, hence it is consistent with the gauge theory.

As discussed in details in Kambe (2020, $§ 1$ and 3.1), this new formulation introduced four independent fields. In fact, regarding the 3 -vector potentials $\boldsymbol{U}$ and $\boldsymbol{Z}$, each has three components. Those six fields have two invariance conditions of (1.7), i.e. two divergence-free conditions in 3-space. In addition, from (1.8) and the equation, $\left(\mathcal{L}_{t}^{*}[\boldsymbol{U}]\right)_{i} \equiv \partial_{t} U_{i}+v^{k} \partial_{k} U_{i}+U_{k} \partial_{i} v^{k}=0$ obtained from the variational analysis of Kambe (2013), we have the third invariance condition:

$$
\mathrm{D}_{t}\langle\boldsymbol{U}, \boldsymbol{Z}\rangle(t, \boldsymbol{x}) \equiv\left\langle\mathcal{L}_{*}[\boldsymbol{U}], \boldsymbol{Z}\right\rangle+\langle\boldsymbol{U}, \mathcal{L}[\boldsymbol{Z}]\rangle=0 .
$$

Hence, the value of scalar product $\langle\boldsymbol{U}, \boldsymbol{Z}\rangle$ is invariant along the particle path $\boldsymbol{x}=$ $\boldsymbol{X}_{p}(t, \boldsymbol{x})$, keeping its initial value along each trajectory. This is the third invariant imposed on the potentials $\boldsymbol{U}$ and $\boldsymbol{Z}$. Therefore we have only three independent fields remaining free among the six components of $\boldsymbol{U}$ and $\boldsymbol{Z}$. Furthermore, if we add the scalar field $\psi$ which is also unconstrained, we have four independent fields in this solution.

Thus, four independent background fields are newly introduced in this solution. Those must be either given externally or determined internally within the framework of theory. In the recent study Kambe (2020), the latter approach was taken, and the general solution of Kambe (2013) is understood to predict existence of new fields $\tilde{a}_{\nu}$. Four independent fields $\tilde{a}_{\nu}$ existing in the $4 d$-spacetime enables a gauge-theoretic formulation in terms of one-form. On the basis of this perspective, the present study proposes a set of new fields to be introduced according to the gauge principle, which may be called a fluid gauge theory.

Another perspective is as follows. What is the hint to resolve the riddle mentioned in the part c) is as follows. We rewrite the part of action $S^{(\mathrm{g}-\mathrm{inv})}$ of $(1.5)$ as $S^{(\mathrm{int})} \equiv \int \rho \mathrm{d} \mathcal{V} \int \Lambda_{\mathrm{Gi}} \mathrm{d} t$, since this term is considered to describe interaction between the flow-current $j^{\nu}$ and background vector-potentials $\boldsymbol{U}$ and $\boldsymbol{Z}$, and $\boldsymbol{\psi}$. Corresponding to the new name $S^{(\text {int })}$, we rename the scalar product $\langle\boldsymbol{U}, \boldsymbol{Z}\rangle$ with $W$, and newly define a 4-current $j^{\nu}$ and a background field $\tilde{a}_{\nu}$ as

$$
j^{\nu} \equiv(\rho c, \rho \boldsymbol{v})=c \bar{\rho} u^{\nu}, \quad \tilde{a}_{\nu} \equiv-\partial_{\nu}(\psi+W) .
$$

(See (A.3), (B.9) for the definition of $\bar{\rho}, u^{\nu}$.) The interaction part $S^{(\mathrm{int})}$ is expressed by

$$
\begin{gathered}
S^{(\mathrm{int})}=-\iint\left(\rho \mathrm{D}_{t} \psi+\rho \mathrm{D}_{t} W\right) \mathrm{d} \mathcal{V} \mathrm{d} t=\iint j^{\nu} \tilde{a}_{\nu} \mathrm{d} \mathcal{V} \mathrm{d} t, \\
j^{\nu} \partial_{\nu}=\rho\left(\partial_{t}+\boldsymbol{v} \cdot \boldsymbol{\nabla}\right)=\rho \mathrm{D}_{t},
\end{gathered}
$$

where $\partial_{\nu} \equiv \partial / \partial x^{\nu}=\left(c^{-1} \partial_{t}, \nabla\right)$. The 4-current $j^{\nu}$ is defined by

$$
j^{\nu}=\rho v^{\nu}=\rho(c, \boldsymbol{v})=\rho\left(\mathrm{d} X_{p}^{\nu} / \mathrm{d} t\right), \quad \mathrm{d} X_{p}^{\nu}=\left(c \mathrm{~d} t, \mathrm{~d} \boldsymbol{X}_{p}\right)=(c, \boldsymbol{v}) \mathrm{d} t .
$$

where $\mathrm{d} X_{p}^{\mu}$ is 4 -spacetime notation of displacement of a fluid particle $p$, and $\mathrm{d} \boldsymbol{X}_{p}=\boldsymbol{v} \mathrm{d} t$ is 3-space displacement of the particle $p$ moving with 3-velocity $\boldsymbol{v}$ during an infinitesimal time interval $\mathrm{d} t$. Denoting $\Psi \equiv \psi+W$, the field $\tilde{a}_{\nu}$ is given by

$$
\tilde{a}_{\nu}=-\partial_{\nu} \Psi
$$


is analogous to the particular field $\tilde{A}_{\mu}=\partial_{\mu} \Theta$ considered in the recent review paper (Kambe 2021a, Section I b), where all the fields $\boldsymbol{E}$ and $\boldsymbol{B}$ vanish identically. In other words, those fields $\boldsymbol{E}$ and $\boldsymbol{B}$ are potentially existing, but vanish in the particular form of $\tilde{A}_{\mu}=\partial_{\mu} \Theta$. Same can be said that our new potential field $\tilde{a}_{\nu}$ does exist. But with the particular form $\tilde{a}_{\nu}=-\partial_{\nu} \Psi$, the potentially existing new field does not show in observable world. From this observation, new Fluid Gauge Theory is proposed in this paper, according to the theory of general gauge fields proposed by Utiyama $(1956,1987)$.

In particular, the following is important in the context of our problem. Gauge invariance applied to the action $S^{\text {(int) }}$ yields the current conservation law:

$$
\partial_{\nu} j^{\nu}=\partial_{\nu}\left(c \bar{\rho} u^{\nu}\right)=\left(c^{-1} \partial_{t}, \boldsymbol{\nabla}\right) \cdot(\rho c, \rho \boldsymbol{v})=\partial_{t} \rho+\nabla \cdot(\rho \boldsymbol{v})=0 .
$$

(See (2.24) in §2.5.) This equation is understood showing a potentiality of the fluid gauge theory. It is remarkable that the two scalar products, $\partial_{\nu} j^{\nu}$ and

$$
j^{\nu} \partial_{\nu}=c \bar{\rho} u^{\nu} \partial_{\nu}=(\rho c, \rho \boldsymbol{v}) \cdot\left(c^{-1} \partial_{t}, \boldsymbol{\nabla}\right)=\rho\left(\partial_{t}+\boldsymbol{v} \cdot \nabla\right) \equiv \rho \mathrm{D}_{t},
$$

are represented relativistically, and that both of them are invariant with respect to the Lorentz transformation. Thus the above two expressions (1.15) and (1.16) show us a glimpse of structures of linked $4 \mathrm{~d}$-spacetime existing in fluid mechanics.

\section{e) A motivation for fluid gauge theory}

From these observations, we set out toward a new approach of fluid gauge theory.

i. Gauge transformations and gauge principle: In the gauge principle, the system under investigation is examined whether the system is invariant with respect to both global and local transformations. The global gauge transformation is defined by the transformation: $\tilde{a}_{\mu} \rightarrow a_{\mu}=\tilde{a}_{\mu}+\epsilon_{\mu}$ for $\tilde{a}_{\mu}$ of (1.14) and $\epsilon_{\mu}$ being 4 arbitrary constants. Firstly, the system must be shown to be invariant with this global transformation. This is the first step toward the fluid gauge theory, examining whether the system under consideration is equipped with desirable receptive property.

A next essential step of the gauge principle lies in requiring local gauge invariance. This is defined by $\tilde{a}_{\mu} \rightarrow a_{\mu}=\tilde{a}_{\mu}+\alpha_{\mu}\left(x^{\nu}\right)$ for 4 arbitrary differentiable fields $\alpha_{\mu}\left(x^{\nu}\right)$ depending on spacetime coordinates $x^{\nu}$. Once the local invariance is established, the so-called gauge-potential $a_{\mu}$ is taken into the system which represents a new interaction force. This is the scenario of the gauge principle to introduce a new force into the system under consideration by the local gauge invariance.

ii. Important factor for the gauge theory: From the gauge principle and reflecting on the form of the interaction action $S^{(\mathrm{int})}$ of $(1.11)$, one realizes that an important factor is the linked $4 d$-spacetime structure. In order to see it, let us remind of the general solution of Euler's equation of motion considered in the part d). This corresponds to a vanishing-field state, because, considering one-form $\tilde{A}$ defined by $\tilde{A}=\tilde{a}_{\mu} \mathrm{d} x^{\mu}$ with $\tilde{a}_{\nu}=-\partial_{\nu} \Psi$ of (1.14), one obtains

$$
\tilde{A}=\tilde{a}_{\mu} \mathrm{d} x^{\mu}=-\partial_{\mu} \Psi \mathrm{d} x^{\mu}=-\mathrm{d} \Psi .
$$

This represents the vanishing-field state since $\mathrm{d} \tilde{A}=-\mathrm{d}^{2} \Psi \equiv 0$. This is the case of the general solution to the Euler's equation of the part d) The field $\tilde{a}_{\mu}$ itself exists, but does not show in the observable world (see Section II, e) ii). Only the Euler's flow field is observed. In this case, the stress field is given by the isotropic pressure field $p \delta_{i k}$, 
On the other hand, existence of the new field $a_{\mu}\left(x^{\nu}\right)=\tilde{a}_{\mu}+\alpha_{\mu}\left(x^{\nu}\right)$ changes the flow field drastically. Consider the $4 \mathrm{~d}$-spacetime $\left(x^{\nu}\right)$ of fluid flows that is structured with the one-form $\mathcal{A} \equiv a_{\mu} d x^{\mu}$, from which one obtains non-vanishing field strength, $\mathcal{F}=\mathrm{d} \mathcal{A} \neq 0$. This gives rise to anisotropic stress field within the flow field, as given in later sections (see Sections III, c) ii.). Thus, the main factor is the one-form defined by

$$
\mathcal{A}=a_{\mu} d x^{\mu}
$$

that plays the role of a game-changer from vanishing-field state of $\tilde{a}_{\mu}$ to non-vanishing state of the new field $a_{\mu}\left(x^{\nu}\right)$. With this fact, the gauge principle is rooted on the fundamental of Physics. This is the central theme of the present paper.

\section{il. An Approach Aiming at a Fluid Gauge Theory}

\section{a) Euler's equation of a perfect fluid in the absence of background field}

Relativistic form of the action integral of a perfect fluid is given in Appendix B.2 as

$$
S^{(\mathrm{pf})}=-c \int \rho \mathrm{d} \mathcal{V} \int\left(1+c^{-2} \bar{\epsilon}(\rho)\right) \mathrm{d} \tau
$$

where $\tau$ the proper time. Its increment $\mathrm{d} \tau$ is defined by the time increment (multiplied by $c$ ) in the instantaneously rest frame where $\boldsymbol{v}=0$. The relativistic action $S^{(\mathrm{pf})}$ is defined as an extension to the perfect fluid from that of a single particle of mass $m$ represented by $S^{(\mathrm{m})}=-c m \int \mathrm{d} \tau$, which is given in Appendix B.1. The overlined $\bar{\epsilon}$ in (2.1) denotes proper value of the internal energy $\epsilon$ (the value in the rest frame, i.e. comoving frame where the fluid is at rest). Comparing $S^{(\mathrm{pf})}$ with $S^{(\mathrm{m})}$ and considering the quantity $\int \rho \mathrm{d} \mathcal{V}$ corresponding to the mass $m$ of $S^{(\mathrm{m})}$, one can see that the second correction term $c^{-2} \bar{\epsilon}$ in the parenthesis is a small correction for the fluid medium in non-relativistic case.

Non-relativistic limit (as $\beta \rightarrow 0$, Section I, c) of the integrand $\Lambda_{(\mathrm{pf})}$ of $S^{(\mathrm{pf})}$ (multiplied by $c)$ per unit mass is given as $\Lambda^{(\mathrm{pf})}=-m_{1} c^{2}+\frac{1}{2} m_{1} v^{2}-\epsilon+\cdots$ with $m_{1}=1$. Neglecting the first term $m_{1} c^{2}$ of the rest-mass energy, the Lagrangian density $\Lambda_{(\mathrm{pf})}$ reduces to the non-relativistic form of $\Lambda_{(\mathrm{nR})}$ of (1.6). Hence it is seen that the action $S^{(\mathrm{pf})}$ is a relativistic version extended from the classic non-relativistic action $S^{(\mathrm{nR})}$ of $(1.5)$.

From the variation analysis of $S^{(\mathrm{pf})}$ carried out in Appendix B.2, the action principle yields the following Euler's equation of motion (B.17) as a geodesic equation:

$$
\mathrm{D}_{t} v^{k}+\rho^{-1} \partial_{k} p=0, \quad k=1,2,3
$$

in the non-relativistic limit of ordinary fluid flows. Noting that the factor $\partial_{k} p$ of the second term can be replaced by $\partial_{j}\left(p \delta_{j k}\right)$, one can rewrite the equation $(2.2)$ as

$$
\rho \mathrm{D}_{t} v^{k}=-\partial_{j}\left(p \delta_{j k}\right) \quad k=1,2,3
$$

where

$$
\mathrm{D}_{t} \equiv \partial_{t}+\boldsymbol{v} \cdot \nabla \quad k=1,2,3
$$

is the convective derivative, which is invariant with respect to local gauge transformation (Kambe (2020), Appendix A2). 


\section{b) New action $S^{(\text {int) }}$ including a field $a_{\mu}$ ensuring current conservation}

In the local gauge transformation considered in Section I, e) i. the field $\alpha_{\mu}$ is assumed to take a general form not limited to the particular gradient form $\partial_{\mu} \Psi$, i.e. not like (1.10), the interaction action $S^{(\mathrm{int})}$ of (1.11) should be extended to general 4-potential $a_{\mu}$ by replacing the particular field $\tilde{a}_{\mu}$. Hence now, the action $S^{(\text {int })}$ is re-defined by

$$
S^{(\text {int })}=\iint j^{\mu} a_{\mu} \mathrm{d} \mathcal{V} \mathrm{d} t=\int \rho \mathrm{d} \mathcal{V} \int v^{\mu} a_{\mu} \mathrm{d} t=\int \rho \mathrm{d} \mathcal{V} \int a_{\mu} \mathrm{d} X^{\mu},
$$

where $j^{\nu}=\rho v^{\mu}$ and see (1.13). This action $S^{(\mathrm{int})}$ was already introduced in Kambe (2020) at its $\S 4.2$. This is rewritten here as an additional action to be added to the main part $S^{(\mathrm{pf})}$, in order to constrain the conservation of current $j^{\mu}$ :

$$
S^{(\text {int })}=\iint j^{\nu} a_{\nu} \mathrm{d} \mathcal{V} \mathrm{d} t=\int \rho \mathrm{d} \mathcal{V} \int a_{\nu} \mathrm{d} x^{\nu}
$$

The one-form structure $a_{\nu} \mathrm{d} x^{\nu}$ in the last integral reminds us of the similar structure considered in the previous section I, e) ii. Similar structure is known in quantum electrodynamics (Section II, b) of Kambe (2021a)). There, the wave function is required to undergo the transformation $\psi=\psi_{0}\left(x^{\nu}\right) \exp \left[i \gamma \int A_{\nu}\left(x^{\nu}\right) d x^{\nu}\right]$ in the presence of electromagnetic field of 4-potential $A_{\nu}$ from the zero-field wave function $\psi_{0}$, where the $A_{\nu}$ field is the gauge-potential representing a new interaction force of electromagnetism.

Our case is based on the gauge principle such that the action $S^{(\mathrm{int})}$ thus introduced represents the interaction between the current field $j^{\nu}\left(x^{\lambda}\right)$ and a background (gaugepotential) field $a_{\nu}\left(x^{\lambda}\right)$ and is receptive to the gauge principle requiring local gauge invariance. The new field $a_{\nu}\left(x^{\nu}\right)$ thus introduced ensures the mass conservation (2.25) shown in Section II, e) iii. under the requirement of gauge invariance of the new field.

In addition, in the Maxwell system described in Section II, a) i. of the review article (Kambe 2021a), the interaction action is given by $S_{\text {int }}^{(\mathrm{em})}=c^{-2} \int j_{e}^{\nu} A_{\nu} \mathrm{d} \Omega$ of eq.(2.9) of the same article. Amazingly, the analogy with the present system is obvious.

\section{c) Composite action $S_{c}$ and modified Euler's equation of motion}

According to the previous sections II a) and b), one can define a composite action $S_{c}$ by using the action $S^{(\mathrm{pf})}$ of a perfect fluid of $(2.1)$ and the action $S^{(\mathrm{int})}$ of (2.6) for the interaction of $j^{\nu}$ and $a_{\nu}$. Let us define

$$
\begin{aligned}
& S_{c} \equiv S^{(\mathrm{pf})}+S^{(\mathrm{int})}, \quad \mathrm{d} \Omega \equiv \mathrm{d}^{4} x=\mathrm{d} \mathcal{V} \mathrm{d} t_{c}, \quad t_{c}=c t, \\
& S^{(\mathrm{pf})} \equiv-c \int \rho \mathrm{d} \mathcal{V} \int\left(1+c^{-2} \bar{\epsilon}(\rho)\right) \mathrm{d} \tau=\int \mathcal{L}^{(\mathrm{pf})} \mathrm{d}^{4} x, \\
& S^{(\text {int })} \equiv \int \mathcal{L}^{\text {(int) }} \mathrm{d}^{4} x, \quad \mathcal{L}^{\text {(int) }} \equiv c^{-1} j^{\nu} a_{\nu},
\end{aligned}
$$

where $\mathcal{L}^{(\mathrm{pf})} \equiv-c \rho\left(1+c^{-2} \bar{\epsilon}\right) \sqrt{1-\beta^{2}}$. To find the equations of motion, the action principle is applied to the composite action $S_{c}$, by assuming the gauge potential $a_{\nu}$ given and vary only the position coordinate $X_{p}^{k}$ of fluid particles moving with the velocity $\mathrm{D}_{t} X_{p}^{k}$ along their trajectories. On the other hand, to find the equations governing the $a_{\nu}$, we vary only the gauge-potential $a_{\nu}$ with assuming the fluid motion given and fixed. However, to carry out the latter variation, we have to define a third action to characterize the background field $a_{\nu}$ and add it to $S_{c}$. Here, we carry out the former variation (in which the third action is kept fixed), then the action principle applied to the varied $S_{c}$ should yield the equation of fluid motion. 
Note that, under the requirement of invariance of $S^{(\mathrm{int})}$ to the gauge transformation of potential $a_{\nu}$ considered in Section II, e), the current conservation law $\partial_{\nu} j^{\nu}=0$ is deduced. Therefore, when variations are taken with respect to the particle position $X_{p}^{k}$, the invariance of the mass $\mathrm{d} m \equiv \rho \mathrm{d} \mathcal{V}$ is assumed for a fluid particle during the motion along its trajectory.

Modified Euler's equation of motion in the presence of new field $a_{\nu}$

The variational analysis of the composite action $S_{c}$ is given in Appendix B.3. The equation (B.26) summarizes the variation analyses with respect to the particle coordinate $\delta X^{\nu}$ of fluid element $\Delta m$,

$$
\delta \mathcal{J}^{(\mathrm{f}+\mathrm{a})} \equiv \delta \mathcal{J}^{(\mathrm{pf})}+\delta \mathcal{J}^{(\mathrm{int})}=-c \overline{\Delta m}\left[\frac{\mathrm{d}}{\mathrm{d} \tau} u_{\nu}+c^{-2} \frac{1}{\bar{\rho}} \partial_{\nu} \bar{p}-c^{-1} f_{\nu \mu} u^{\mu}\right] \mathrm{d} \tau \delta X^{\nu}
$$

as leading order terms in the expansion with respect to the very small parameter $\beta=v / c$. The action principle requires $\delta \mathcal{J}^{(\mathrm{f}+\mathrm{a})}=0$ for arbitrary variation $\delta X^{\nu}$. This leads to the equation: $\left(\mathrm{d} u_{\nu} / \mathrm{d} \tau\right)+c^{-2}(1 / \bar{\rho}) \partial_{\nu} \bar{p}-c^{-1} f_{\nu \mu} u^{\mu}=0$. Its non-relativistic limit $($ as $\beta \rightarrow 0)$ is expressed by the equations:

$$
\begin{gathered}
\mathrm{D}_{t} v^{k}=-\rho^{-1} \partial_{k} p+f_{k \nu} v^{\nu}, \quad(k=1,2,3 ; \quad \nu=0,1,2,3), \\
f_{\mu \nu}=\partial_{\mu} a_{\nu}-\partial_{\nu} a_{\mu} .
\end{gathered}
$$

This is the Euler's equation (2.2) modified by the effect of a background field $a^{\nu}$ expressed by the third term $f_{k \nu} v^{\nu}$. Section III c), ii. gives details concerning the significance of the new tensor field $f_{\mu \nu}$ by the section title, Background field: Fluid Maxwell fields.

\section{d) Scenario of general gauge principle according to Utiyama}

In the previous section II c), we have derived the modified Euler equation (2.11) from the composite action $S_{c} \quad\left(=S^{(\mathrm{pf})}+S^{(\mathrm{int})}\right)$ of $(2.7)$. The first term $-\rho^{-1} \partial_{k} p$ on the righthand side of (2.11) represents the pressure force caused by the isotropic stress tensor $-p \delta_{k l}$, while the second term $f_{k \nu} v^{\nu}$ represents a new force caused by an anisotropic stress field, as explained in a later section III c), iii. It is remarkable that the tensor components $f_{\mu \nu}$ of (2.12) all are linear with respect to space-time derivatives of the background potential $a_{\mu}$. This is essential for the gauge principle to be given now.

Suppose that our basic undisturbed state is described by the equation (2.11) and by the composite action $S_{c}$ of (2.7), and in addition that the background field $a_{\mu}$ is given a special form, i.e. a potential field expressed by $\tilde{a}_{\mu}=\partial_{\mu} \Psi$ with $\Psi\left(x^{\nu}\right)$ a scalar field. Note that this field form $\tilde{a}_{\mu}=\partial_{\mu} \Psi$ is a special class among general background fields.

As described in the next section II e), ii. in details, all the components $f_{\mu \nu}$ associated with the background potential $\tilde{a}_{\mu}=\partial_{\mu} \Psi$ vanish identically. Hence the equation (2.11) reduces to the Euler's equation (2.2). Namely, the basic undisturbed state is assumed to be governed by the Euler's equation. In addition, from the action $S^{\text {int }}$, the continuity equation (2.14) was derived in II e), iii.

In this case, the action $S^{(\text {int })}$ does not give any mechanical effect on the system (see II e), ii.). Therefore, the mechanical effect of the composite action $S_{\mathrm{c}}$ is equivalent to that of the term $S^{(\mathrm{pf})}$ of perfect fluid only, not different from the state without the field $a_{\mu}$. Therefore, for the potential fields of $\tilde{a}_{\mu}=\partial_{\mu} \Psi$, the basic undisturbed state is represented by that of the perfect-fluid action $S^{\text {pf }}$ only. Namely, the state is nothing but the Euler field.

According to Utiyama $(1956,1987)$, the general gauge principle states as follows. "If both of the composite action $S_{c}$ and the equation of motion (2.11) are invariant under 
a global transformation defined by $\tilde{a}_{\mu} \rightarrow \tilde{a}_{\mu}^{\prime}=\tilde{a}_{\mu}+\delta a_{\mu}$ for uniformly constant value of $\delta a_{\mu}=\epsilon_{\mu}$, then the system is said invariant globally for the $a^{\mu}$-transformation."

The invariance of the governing equation (2.11) is almost trivial because the field $\tilde{a}_{\mu}$ is included only in $f_{\mu \nu}$ where all the components $\tilde{a}_{\mu}$ are expressed in derivative forms, as seen from (2.12). Hence, constant variation $\epsilon_{\mu}$ of $\tilde{a}_{\mu}$ does not give any effect on the equation (2.11). Concerning the action $S^{(\mathrm{int})}$, its invariance by the global transformation $\tilde{a}_{\mu} \rightarrow \tilde{a}_{\mu}+\epsilon_{\mu}$ is investigated in the section III b) below the line (3.11), and the system is invariant mechanically with this global transformation. Thus, the system is globally invariant for the uniform $\tilde{a}_{\mu}$-variation.

The gauge principle reads furthermore, "Even if the global invariance of the system is satisfied, one may consider local transformation with $\delta a_{\mu}\left(x^{\nu}\right)$ varying with the spacetime coordinates $x^{\nu}$, for which neither the action integral nor the equation of motion are invariant locally under such a local gauge transformation."

Here, we have to remind that the interaction action $S^{(\mathrm{int})}$ of $(2.9)$ is already defined by using general $a_{\mu}\left(x^{\nu}\right)$ field depending on the space-time coordinates $x^{\nu}$. In the previous Section II c) using this $S^{(\text {int })}$, we have derived the equation of motion (2.11). For the particular form of potential $\tilde{a}_{\mu}=\partial_{\mu} \Psi$ considered above in the global transformation, the new tensor field $f_{\mu \nu}$ vanishes identically (verified immediately by substitution). Then, the equation (2.11) reduces to the Euler's equation (2.2). Under the local transformation, however, the tensor field $f_{\mu \nu}$ does not vanish in general, then the equation (2.11) deviates from the Euler's equation (2.2). In other words, the equation (2.11) is not invariant for the local transformation: $a_{\mu} \rightarrow a_{\mu}+\delta a_{\mu}\left(x^{\nu}\right)$. This fact is interpreted as follows.

There may exist a background field $a_{\mu}$ in the flow field $v^{\mu}\left(x^{\nu}\right)$, which interacts with the flow under the action of the stress field $f_{\mu \nu} v^{\nu}$. The last is a new stress field. Thus, the general gauge principle predicts existence of a certain background field $a_{\mu}$ and an internal stress field $f_{\mu \nu} v^{\nu}$ generated by $a_{\mu}$. The original basic state was the one governed by the Euler's equation. The equation (2.3) states that its stress field is given by an isotropic stress tensor $-p \delta_{i k}$. In the present context, corresponding equation (2.11) can be rewritten as

$$
\rho \mathrm{D}_{t} v^{k}=-\partial_{j}\left(p \delta^{j k}\right)+\rho f_{\nu}^{k} v^{\nu},(k=1,2,3 ; \quad \nu=0,1,2,3),
$$

Later in section III c) ii. the second term on the right $\rho f_{\nu}^{k} v^{\nu}$ is rewritten by (3.24) as $-\partial_{\nu} M^{\nu k}$, which represents anisotropic stress field.

It is essential in the scenario of the general gauge principle of Utiyama that the background field $a_{\mu}$ ensures the current conservation $\partial_{\mu} j^{\mu}=0$, by the gauge invariance property of the background field $a_{\mu}$ itself. In the author's previous paper (Kambe, 2020), this action $S^{(\mathrm{int})}$ was already introduced at its section 4.2 " Gauge invariance and mass conservation", where invariance of $S^{(\mathrm{int})}$ was required to the gauge transformation $a_{\mu} \rightarrow a_{\mu}-\partial_{\mu} \Psi_{*}$ for arbitrary scalar field of $\Psi_{*}\left(x^{\nu}\right)$. The close connection between the gauge invariance and the law of mass conservation has been established there.

In the next section II e) iii. requiring the invariance of the action $S^{(\mathrm{int})}$ under the gauge transformation for arbitrary scalar field of $\Psi\left(x^{\nu}\right)$, the mass conservation equation is deduced:

$$
\partial_{\mu} j^{\mu}=\partial_{t} \rho+\nabla \cdot(\rho \boldsymbol{v})=0 .
$$

This is the scenario of the general gauge principle of Utiyama.

According to the scenario of Utiyama's general gauge principle, we have to show one more, which is as follows. The new field $a_{\mu}$ should be incorporated as a connection term in a covariant derivative. This is done in the Appendix B.4 where physical and mathematical formulations for curved space are presented and applied to flow fields of a perfect fluid. 
This enables to introduce a general notion of covariant derivatives (connections). Its mathematical formulation is applied to free evolution of physical systems (free from external actions) but exhibiting non-straight motion even in flat space. Mathematical formulation by geometrical language enables us to formulate this generalization, namely enabling to conclude the fluid gauge theory.

e) Background field $a_{\nu}$ ensuring current conservation $\partial_{\mu} j^{\mu}=0$

i. Background field $a_{\nu}$ represented by $\mathcal{A}, \mathcal{F}$ and $\boldsymbol{f}_{a}$

Using the background field $a_{\nu}$, one can define a one-form $\mathcal{A} \equiv a_{\nu} \mathrm{d} x^{\nu}$. Taking its exterior differential $\mathrm{d}$, a field strength two-form $\mathcal{F}$ is given by

$$
\mathcal{F} \equiv \mathrm{d} \mathcal{A}=\sum_{\nu<\lambda} f_{\nu \lambda} \mathrm{d} x^{\nu} \wedge \mathrm{d} x^{\lambda}, \quad f_{\nu \lambda} \equiv \partial_{\nu} a_{\lambda}-\partial_{\lambda} a_{\nu}=-f_{\lambda \nu}
$$

where $\partial_{\nu} \equiv \partial / \partial x^{\nu}=\left(c^{-1} \partial_{t}, \boldsymbol{\nabla}\right)$, the tensor $f_{\nu \lambda}$ is anti-symmetric.

Representing the 4-potential $a_{\nu}$ with a $(1+3)$-expression given by $a_{\nu}=(-\phi / c, \boldsymbol{a})$ where $a_{0}=-\phi / c$ and $\boldsymbol{a}=\left(a_{k}\right)=\left(a_{1}, a_{2}, a_{3}\right)$, one can define

$$
\begin{aligned}
& \boldsymbol{b}=\left(b_{k}\right) \equiv \nabla \times \boldsymbol{a} ; \quad f_{i j}=\partial_{i} a_{j}-\partial_{j} a_{i}=\varepsilon_{i j k} b_{k}, \quad b_{k}=\varepsilon_{k l m} \partial_{l} a_{m} \\
& \boldsymbol{e}=\left(e_{k}\right) \equiv-\partial_{t} \boldsymbol{a}-\nabla \phi ; \quad f_{k 0}=\partial_{k} a_{0}-\partial_{0} a_{k}=c^{-1} e_{k} \equiv \bar{e}_{k} .
\end{aligned}
$$

where each of $i, j$ and $k$ takes the number of either 1,2 , or 3 .

The modified Euler equation (2.11) includes the term of internal stress field $f_{\mu \nu} v^{\nu}$, which can be given a 3-vector form by using 3-vectors $\boldsymbol{e}, \boldsymbol{b}$ and $\boldsymbol{v}$ as

$$
\begin{aligned}
& \boldsymbol{f}_{a}=\left(f_{a, i}\right) \equiv \boldsymbol{e}+\boldsymbol{v} \times \boldsymbol{b}, \quad f_{a, i}=f_{i \nu} v^{\nu}=f_{i 0} v^{0}+f_{i k} v^{k} . \\
& f_{a, i}=f_{i \nu} v^{\nu}=f_{i 0} v^{0}+f_{i j} v^{j}=c^{-1} e_{i} c+\left(\varepsilon_{i j k} b_{k}\right) v^{j}=(\boldsymbol{e}+\boldsymbol{v} \times \boldsymbol{b})_{i},
\end{aligned}
$$

where $v^{\nu}=\left(v^{0}, \boldsymbol{v}\right)=(c, \boldsymbol{v})$ from $(1.13)$.

ii. Special background field $\tilde{a}_{\mu}=\partial_{\mu} \Psi$ implies Eulerian field

Using $\tilde{a}_{\mu}=\partial_{\mu} \Psi$, let us define a one-form by $\tilde{A}=\tilde{a}_{\nu} \mathrm{d} x^{\nu}=\left(\partial_{\nu} \Psi\right) \mathrm{d} x^{\nu}=\mathrm{d} \Psi$. Then the field strength two-form $\tilde{\mathcal{F}} \equiv \mathrm{d} \tilde{A}$ vanishes identically since $\mathrm{d}^{2} \Psi \equiv 0$, which can be shown alternatively by using the vanishing components $f_{\nu \lambda}$ :

$$
\tilde{\mathcal{F}}=\mathrm{d} \tilde{A}=\sum_{\nu<\lambda} f_{\nu \lambda} \mathrm{d} x^{\nu} \wedge \mathrm{d} x^{\lambda} \equiv 0, \quad \text { i.e. } \quad f_{\nu \lambda}=\partial_{\nu} \partial_{\lambda} \Psi-\partial_{\lambda} \partial_{\nu} \Psi=0 .
$$

Hence, the background field of the type $\tilde{a}_{\mu}=\partial_{\mu} \Psi$ constitutes a special class in the flow field, and that, for this type of field, the modified Euler equation (2.11) reduces to the original Euler's equation (2.2), because all the fields $f_{\mu \nu}$ derived from $\tilde{a}_{\mu}=\partial_{\mu} \Psi$ vanish identically. In other words, the tensor fields $f_{\mu \nu}$ are potentially existing, but vanish for the particular form $\tilde{a}_{\mu}=\partial_{\mu} \Psi$, where $\Psi\left(x^{\nu}\right)$ is an arbitrary twice differentiable scalar field in the spacetime $x^{\nu}$. The $\tilde{a}_{\mu}$ field does exist, but with the particular form $\tilde{a}_{\nu}=\partial_{\nu} \Psi$, the potentially existing fields do not show in observable world. Thus, the potential form, $\tilde{a}_{\mu}=\partial_{\mu} \Psi$, constitutes a special class of background field.

Hence, the modified Euler equation (2.11) reduces to the original Euler equation (2.2). In addition, regarding the composite action $S_{c}=S^{(\mathrm{pf})}+S^{(\mathrm{int})}$, the interaction part $S^{(\text {int })}$ does not give any mechanical effect on the system with the special field $\tilde{a}_{\mu}=\partial_{\mu} \Psi$. The field $\tilde{a}_{\mu}$ is included only in the interaction action $S^{(\mathrm{int})}$ of $(2.9)$.

The variational principle requires that variation of the action $S_{c}$ must vanish with respect to the variation $\tilde{a}_{\mu}$ given by $\delta \tilde{a}_{\mu}=\partial_{\mu}(\delta \Psi)$ where $\delta \Psi$ is the variation 
of $\Psi$. Substituting the $\tilde{a}_{\mu}$-variation into $S^{(\mathrm{int})}$, its resulting variation $\delta S^{(\mathrm{int})}$ owing to $\delta \tilde{a}_{\mu}=\partial_{\mu}(\delta \Psi)$ is given by

$$
\delta S^{(\mathrm{int})}=\int j^{\mu}\left(\partial_{\mu} \delta \Psi\right) \mathrm{d} \Omega=-\int\left(\partial_{\mu} j^{\mu}\right) \delta \Psi \mathrm{d} \Omega+\int \partial_{\mu}\left(j^{\mu} \delta \Psi\right) \mathrm{d} \Omega,
$$

where $\mathrm{d} \Omega=\mathrm{d} \mathcal{V} \mathrm{d} t$. The invariance requires the integral on the left-hand side to vanish. The last integral of 4-divergence $\partial\left(\Psi j^{\nu}\right) / \partial x^{\nu}$ on the right-hand side is transformed to vanishing integrals over bounding hypersurfaces (where the imposed function $\Psi$ is assumed to vanish, or irrelevant because the variational analysis is carried out only at internal points). Vanishing of the first integral for arbitrary variation $\delta \Psi$ (at internal points) leads to the following equation of the mass conservation:

$$
\partial_{\nu} j^{\nu}=\partial_{\tau}\left(\rho c_{*}\right)+\partial_{k}(\rho \boldsymbol{v})_{k}=\partial_{t} \rho+\nabla \cdot(\rho \boldsymbol{v})=0 .
$$

Thus, the mechanical effect of the composite action $S_{c}$ reduces to that of the term $S^{\text {(pf) }}$ of perfect fluid without the background field $a_{\mu}$. Therefore, for potential fields of $\tilde{a}_{\mu}=\partial_{\mu} \Psi$, the basic undisturbed state is equivalent to the Eulerian field constrained with the mass conservation equation.

\section{iii. Gauge invariance of $S^{(\mathrm{int})}$ requires the mass conservation}

It is essential in the scenario of the general gauge principle of Utiyama that the background field $a_{\mu}$ ensures the current conservation $\partial_{\mu} j^{\mu}=0$, by the gauge invariance property of the background field $a_{\mu}$ itself. Likewise the transformation done in Kambe (2021a; section II a), we define a one-form $\mathcal{A} \equiv a_{\nu} \mathrm{d} x^{\nu}$ and introduce an arbitrary scalar field $\Theta\left(x^{\nu}\right)$. Then, we carry out a gauge transformation, $\mathcal{G}: a_{\nu} \rightarrow a_{\nu}^{\prime}=a_{\nu}-\partial_{\nu} \Theta$, and we have

$$
\mathcal{A}^{\prime} \equiv a_{\nu}^{\prime} \mathrm{d} x^{\nu}=\left(a_{\nu}-\partial_{\nu} \Theta\right) \mathrm{d} x^{\nu}=a_{\nu} \mathrm{d} x^{\nu}-\partial_{\nu} \Theta \mathrm{d} x^{\nu}=\mathcal{A}-\mathrm{d} \Theta .
$$

From this, we find the invariance of the field strength two-form $\mathcal{F} \equiv \mathrm{d} \mathcal{A}$ as follows:

$$
\mathcal{F}^{\prime} \equiv \mathrm{d} \mathcal{A}^{\prime}=\mathrm{d} \mathcal{A}+\mathrm{d}^{2} \Theta=\mathrm{d} \mathcal{A} \equiv \mathcal{F},
$$

since $\mathrm{d}^{2} \Theta=0$ identically. Thus it is found that the two-form $\mathcal{F}$ is invariant with respect to the gauge transformation $\mathcal{G}$.

Matrix elements of $\mathcal{F}$ represented by $f_{\nu \lambda}=\partial_{\nu} a_{\lambda}-\partial_{\lambda} a_{\nu}$ are also gauge-invariant. The expressions of (3.15) give matrix-form representations of $f_{\nu \lambda}$ and its contravariant form $f^{\nu \lambda}$. In these matrix forms, the 4-potential $a_{\nu}$ is expressed by $(-\phi / c, \boldsymbol{a})$, together with $\boldsymbol{b}=\left(b_{k}\right) \equiv \nabla \times \boldsymbol{a}$ and $\boldsymbol{e}=\left(e_{k}\right) \equiv-\partial_{t} \boldsymbol{a}-\nabla \phi$. Thus, the background field matrix $f_{\nu \lambda}$ can be represented by components of $\boldsymbol{b}$ and $\boldsymbol{e}$, which are also gauge-invariant.

Next, let us require invariance of the action $S^{(\text {int) }}$ under the gauge transformation $\mathcal{G}$ for arbitrary scalar field of $\Theta\left(x^{\nu}\right)$. By replacing $a_{\mu}$ with $a_{\mu}-\partial_{\mu} \Theta$. Then, the action $S^{\text {(int) }}$ of (2.9) has an additional term (which is required to vanish),

$$
-\int j^{\mu}\left(\partial_{\mu} \Theta\right) \mathrm{d} \Omega=\int\left(\partial_{\mu} j^{\mu}\right) \Theta \mathrm{d} \Omega-\int \partial_{\mu}\left(j^{\mu} \Theta\right) \mathrm{d} \Omega
$$

The gauge invariance requires the integral on the left-hand side to vanish. The last integral of 4-divergence $\partial\left(\Theta j^{\nu}\right) / \partial x^{\nu}$ is transformed to vanishing integrals over bounding surfaces where the imposed function $\Theta$ is assumed to vanish. Vanishing of the above integral for arbitrary $\Theta$ at internal points leads to the current conservation $\partial_{\mu} j^{\mu}=0$. Namely, the following mass conservation equation must be satisfied:

$$
\partial_{\mu} j^{\mu}=\partial_{t} \rho+\nabla \cdot(\rho \boldsymbol{v})=0 .
$$

Hence, the invariance of $S^{(\text {int })}$ under the transformation $\mathcal{G}$ requires the mass conservation equation to be satisfied. 
iv. Action $S^{(\mathrm{F})}$ of the background Field $a_{\nu}$

Up to now, the action formulation on our fluid system is not completed. To make the fluid system self-contained, we need a third action $S^{(\mathrm{F})}=\int \mathcal{L}^{(\mathrm{F})} \mathrm{d} \Omega$ governing freestate of the background field $a_{\nu}$, describing only on the property of the field itself. To establish the form of the Lagrangian density $\mathcal{L}^{(\mathrm{F})}$ of the field, we start from the following observation and requirements:

(i) The tensor field $f_{\nu \lambda}$ should be ensured to vanish when the background field $a_{\nu}$ takes the special form $\tilde{a}_{\nu}=\partial_{\mu} \Psi$ with $\Psi\left(x^{\nu}\right)$ a twice differentiable scalar field. This means the following. According to the item $(i$.) of this section, the original Euler's equation of motion (2.2) is valid in spite of the existence of the field $a_{\nu}$.

(ii) The Lagrangian density $\mathcal{L}^{(\mathrm{F})}$ is a Lorentz scalar, i.e. invariant with respect to the Lorentz transformation (Appendix).

(iii) In the subsections $(i.) \sim($ iii. . of this section II e) we have already defined the field strength tensor $f_{\mu \nu}=\partial_{\mu} a_{\nu}-\partial_{\nu} a_{\mu}$, which is gauge-invariant and also satisfies the condition $(i)$, namely $f_{\mu \nu}=0$ for $a_{\mu}$ given by $\tilde{a}_{\nu}=\partial_{\mu} \Psi$. This representation of $f_{\mu \nu}$ was derived from the one-form $\mathcal{A}=a_{\mu} d x^{\mu}$ defined by (1.17) in Sections I e) ii.

Under these conditions, we expect the free-Lagrangian $\mathcal{L}^{(\mathrm{F})}$ to be quadratic in $\partial_{\mu} a_{\nu}$ or $f_{\mu \nu}$, because the variation of $S^{(\mathrm{F})}$ reduces the degree by one with resulting equation becoming linear to $\partial_{\mu} a_{\nu}$. The only Lorentz-invariant quadratic form is a multiple of $f_{\mu \nu} f^{\mu \nu}$ (see Landau \& Lifshitz (1975, §27), or Jackson (1999, §12.7), for the corresponding Lagrangian of electromagnetic field).

Thus, the Lagrangian density $\mathcal{L}^{(\mathrm{F})}$ for the background Field $a_{\nu}$ should be represented as

$$
S^{(\mathrm{F})}=\int \mathcal{L}^{(\mathrm{F})} \mathrm{d} \Omega, \quad \mathcal{L}^{(\mathrm{F})} \equiv C f_{\mu \nu} f^{\mu \nu} . \quad C: \text { a constant. }
$$

\section{f) New field equations re-ensuring current conservation}

According to the observations of Sections II c) e) on the actions of the present fluid system, it is proposed that total Lagrangian density $\mathcal{L}$ consists of three terms: Lagrangians of (i) a perfect fluid $\mathcal{L}^{(\mathrm{pf})}$, (ii) a background field $\mathcal{L}^{(\mathrm{F})}$ and $($ iii) their mutual interaction $\mathcal{L}^{\text {(int) }}$. Hence, the total Lagrangian is $\mathcal{L}=\mathcal{L}^{(\mathrm{pf})}+\mathcal{L}^{(\mathrm{int})}+\mathcal{L}^{(\mathrm{F})}$ :

$$
\mathcal{L}^{(\mathrm{pf})}=-c \bar{\rho}\left(1+c^{-2} \bar{\epsilon}\right) ; \quad \mathcal{L}^{(\mathrm{int})}=c^{-1} j^{\mu} a_{\mu} ; \quad \mathcal{L}^{(\mathrm{F})}=-\frac{1}{4 \mu c} f_{\mu \nu} f^{\mu \nu}
$$

where the constant $C$ is rewritten as $C=-(4 \mu c)^{-1}$ with using another constant $\mu$ for later convenience.

i. Action principle

We define the total action $S^{(\text {total })}$ by

$$
S^{(\text {total })}=S_{c}+S^{(\mathrm{F})}=\int\left[\int\left(\mathcal{L}^{(\mathrm{pf})}+\mathcal{L}^{(\mathrm{int})}+\mathcal{L}^{(\mathrm{F})}\right) \mathrm{d} \mathcal{V}\right] c \mathrm{~d} t
$$

where the Lagrangian densities $\mathcal{L}^{(\mathrm{pf})}, \mathcal{L}^{(\mathrm{int})}$ and $\mathcal{L}^{(\mathrm{F})}$ are defined by $(2.27), \mathrm{d} \Omega=c \mathrm{~d} t \mathrm{~d} \mathcal{V}$, $\mathrm{d} \mathcal{V}=\mathrm{d} x^{1} \mathrm{~d} x^{2} \mathrm{~d} x^{3}$ and $\bar{\rho} \overline{\mathrm{d} \mathcal{V}}=\rho \mathrm{d} \mathcal{V} . \dagger$ Relativistic 4-current is defined by $j^{\nu} \equiv \bar{\rho} v^{\nu}$, in addition by

$$
j^{\nu}=\rho \frac{\mathrm{d} X^{\nu}}{\mathrm{d} t}=\rho v^{\nu} \sqrt{1-\beta^{2}}=\rho(c, \boldsymbol{v})
$$

$\dagger \mathrm{d} \tau=c \mathrm{~d} t \sqrt{1-\beta^{2}}, \overline{\mathrm{d} \mathcal{V}}=\mathrm{d} \mathcal{V} / \sqrt{1-\beta^{2}}$ and $\bar{\rho}=\rho \sqrt{1-\beta^{2}}$. Hence $\overline{\mathrm{d} \mathcal{V}} \mathrm{d} \tau=\mathrm{d} \mathcal{V} c \mathrm{~d} t$ 
The tensor $f_{\nu \lambda} \equiv \partial_{\nu} a_{\lambda}-\partial_{\lambda} a_{\nu}$ in the expression $\mathcal{L}^{(\mathrm{F})}$ is a field strength tensor, and $a_{\nu} \equiv(-\phi / c, \boldsymbol{a})$ is a 4 -potential.

To find the equations governing the background field $a_{\nu}$, the principle of least action is applied to the action $S^{\text {(total) }}$. We must assume the fluid motion a given field, hence fixed. We vary only the potential field $a_{\nu}$. In regard to the fluid motion, its equation of motion is already found from the composite action $S_{c}$ in the section II c) where $a_{\nu}$ is assumed to be a given field. The equation of motion is given by (2.11). Citing it,

$$
\mathrm{D}_{t} v^{k}+\rho^{-1} \partial_{k} p-f_{k \nu} v^{\nu}=0, \quad(k=1,2,3 ; \quad \nu=0,1,2,3) .
$$

We aim that the continuity equation of fluid flows is deduced also from this variational analysis. Since the first Lagrangian $\mathcal{L}^{(\mathrm{pf})}$ does not include the field $a_{\nu}$ to be varied, we consider variations of the other two Lagrangians $\mathcal{L}^{(\text {int) }}$ and $\mathcal{L}^{(\mathrm{F})}$.

Variation with respect to $a_{\nu}$

The two Lagrangians $\mathcal{L}^{(\text {int) }}$ and $\mathcal{L}^{(\mathrm{F})}$ include the background field $a_{\nu}$. First, we note $\delta\left(f^{\nu \lambda} f_{\nu \lambda}\right)=2 f^{\nu \lambda}\left(\delta f_{\nu \lambda}\right)$. This is because

$$
\left(\delta f^{\nu \lambda}\right) f_{\nu \lambda}=\left(\delta f^{\nu \lambda}\right) \eta_{\nu \alpha} \eta_{\lambda \beta} f^{\alpha \beta}=f^{\alpha \beta}\left(\delta f_{\alpha \beta}\right) .
$$

See Appendix A for the Minkowski metric $\eta_{\nu \alpha}$.

Therefore, variation of $\mathcal{L}^{(\mathrm{int})}+\mathcal{L}^{(\mathrm{F})}$ is given by

$$
\begin{aligned}
c\left(\delta \mathcal{L}^{(\mathrm{int})}+\delta \mathcal{L}^{(\mathrm{F})}\right)=j^{\nu} \delta a_{\nu}-\frac{1}{2 \mu} f^{\nu \lambda} \delta f_{\nu \lambda} & =j^{\nu} \delta a_{\nu}-\frac{1}{2 \mu} f^{\nu \lambda} \frac{\partial}{\partial x^{\nu}} \delta a_{\lambda} \\
+\frac{1}{2 \mu} f^{\nu \lambda} \frac{\partial}{\partial x^{\lambda}} \delta a_{\nu} & =\left(j^{\nu}-\frac{1}{\mu} \frac{\partial}{\partial x^{\lambda}} f^{\nu \lambda}\right) \delta a_{\nu} .
\end{aligned}
$$

where the term $-(1 / 2 \mu) f^{\nu \lambda} \partial_{\nu}\left(\delta a_{\lambda}\right)$ of the last term on the upper line can be equated to $(1 / 2 \mu) f^{\nu \lambda} \partial_{\lambda}\left(\delta a_{\nu}\right)$ by using the anti-symmetry, $-f^{\nu \lambda}=f^{\lambda \nu}$. On interchanging the indices $\nu$ and $\lambda$, this term can be combined with its next term to give $(1 / \mu) f^{\nu \lambda} \partial_{\lambda}\left(\delta a_{\nu}\right)$. Finally carrying out integration-by-parts leads to the second term of $(2.31)$, with omitting the term of divergence-form $\partial_{\lambda}\left[(1 / \mu) f^{\nu \lambda} \delta a_{\nu}\right]$ which vanishes on integration.

Requiring vanishing of $\delta \mathcal{L}^{(\mathrm{int})}+\delta \mathcal{L}^{(\mathrm{F})}=0$ for arbitrary variation $\delta a_{\nu}$, we obtain

$$
\frac{\partial}{\partial x^{\lambda}} f^{\nu \lambda}=\mu j^{\nu}
$$

where the 4-current $j^{\nu}$ is defined by (2.29). This is the equation governing the background field $f^{\nu \lambda}$ derived from the principle of least action. Thus,

the system of field equations (2.30) and (2.32) have been derived by the invariant variation of the total action $S^{\text {(total) }}$ of (2.28).

\section{ii. Current conservation}

The equation of current conservation can be derived from this, which is directly connected with the gauge-invariant property of the Lagrangian $\mathcal{L}^{(\mathrm{F})}$. This is analogous to the electromagnetic fields (Kambe (2021a) Section II a) ii). In fact, applying the divergence operator $\partial_{\nu}$ on the equation (2.32), one obtains

$$
0=\partial_{\nu} \partial_{\lambda} f^{\nu \lambda}=\mu \partial_{\nu} j^{\nu}
$$

The left-hand side vanishes because of the anti-symmetry of $f^{\nu \lambda}$ and the symmetry of $\partial_{\nu} \partial_{\lambda}$. Total sum with respect to $\nu$ and $\lambda$ (taking indices $0,1,2,3$ ) vanishes identically. Hence, we find the current conservation equation: 


$$
\partial_{\nu} j^{\nu}=0 \quad \Rightarrow \quad \partial_{t} \rho+\nabla \cdot(\rho \boldsymbol{v})=0
$$

for $j^{\nu}=(\rho c, \rho \boldsymbol{v})$. Thus it is found that the newly added Lagrangian $\mathcal{L}^{(\mathrm{F})}$ ensures the mass conservation.

All the analyses, concerning the field potential $a_{\nu}$, the differential forms and the variations, are exactly analogous to the electromagnetic case (Kambe (2021a) §2.1 (a)). Only differences are the letters used, whether those are lower-case or upper-case, and the material constants are different between the two cases. Thus for our fluid system, we obtain the same form of Maxwell-type equations with the field vectors $\boldsymbol{e} \equiv-\partial_{t} \boldsymbol{a}-\nabla \phi$ and $\boldsymbol{b} \equiv \nabla \times \boldsymbol{a}$ with two field constants, $\mu$ and $\varepsilon=1 /\left(c^{2} \mu\right)$ :

$$
\begin{gathered}
\partial_{t} \boldsymbol{b}+\nabla \times \boldsymbol{e}=0, \quad \nabla \cdot \boldsymbol{b}=0 . \\
\varepsilon \nabla \cdot \boldsymbol{e}=\rho, \quad-\varepsilon \partial_{t} \boldsymbol{e}+\mu^{-1} \nabla \times \boldsymbol{b}=\boldsymbol{j} . \\
\text { ili. Fluid Gauge Theory }
\end{gathered}
$$

\section{a) A road to fluid gauge theory}

More than sixty years ago, Utiyama (1956) proposed a general approach to the gauge theory and called it General Gauge Theory. He extended the Weyl's gauge principle (described in Kambe (2021a)) to general Lie groups and included the theory of gravity (O'Raifeartaigh 1997, Chap.10). He realized already the broad analogy between the two theories of gravitational field and electromagnetic field, which is reviewed in Kambe (2021a) too. According to the scenario of the general gauge theory, new fields are introduced in the systems under investigation which are carrying interaction forces such as gravity force or electromagnetic force.

How the fluid-flow field is required to be improved or reformed by the fluid gauge theory ? The answer is that the isotropic pressure stress field is extended to general anisotropic stress field in the flow of perfect fluid if its motion is time-dependent and rotational. This is required by the constraint to the current conservation under background gauge fields. However, the Euler's equation of motion is still valid as far as the stress field is constrained to be isotropic.

On a mathematical point of view, more must be added according to the Utiyama's approach of the General Gauge Theory. The new fields should be taken (or absorbed) into the structure of covariant derivatives as connection terms like the terms of Christoffel symbols in the gravity theory or the gauge potentials in the electromagnetic theory. The concept of connection in the mathematics of Riemannian geometry is an essential ingredient in the physical gauge theory. It is a challenging work to implement connection terms in the structure of fluid gauge theory. This is left to Appendix B (Relativistic formulation of three mechanical systems) and Appendix $\mathrm{C}$ (Free motion of physical systems and curved geodesics), because sufficient mathematical expressions and concepts must be presented to arrive at the goal.

We are going to propose and present a new formulation of Fluid Gauge Theory in this section $\S 3$ by the help of Appendix B and Appendix C. In fact, before giving the final conclusion, we have to examine that free motions are not always described by straight trajectories of time evolution of mechanical systems, namely their geodesics are curved in general. In Appendix C.2, we will see that the free motion of a perfect fluid under a background field $a_{\nu}$ can be described by the geodesic equation representing a curved free dynamics. This is derived by the variational principle that makes the action integrals invariant. Namely the new field of the fluid gauge theory has been taken into the structure of covariant derivatives as connection terms and the free dynamics of a perfect fluid under a background field $a_{\nu}$ is described by the geodesic equation.

Thus, the Fluid Gauge Theory is concluded now. 


\section{b) Fluid Gauge Theory summarized}

According to the scenario of the general gauge principle, the last section II has concluded that the flow field of a perfect fluid is supported with a background field $a_{\mu}$ ensuring current conservation. Now our quest for the fluid gauge theory has come to the final stage.

\section{Statement of the fluid gauge theory:}

Collecting main results obtained in the last section II (An approach aiming at a fluid gauge theory), the fluid gauge theory is presented by the following set of expressions:

$$
\begin{gathered}
S^{(\text {total })}=S^{(\mathrm{pf})}+S^{(\mathrm{int})}+S^{(\mathrm{F})}=\int\left[\int\left(\mathcal{L}^{(\mathrm{pf})}+\mathcal{L}^{(\mathrm{int})}+\mathcal{L}^{(\mathrm{F})}\right) \mathrm{d} \mathcal{V}\right] c \mathrm{~d} t, \\
\mathcal{L}^{(\mathrm{pf})}=-c^{-1}\left(c^{2}+\bar{\epsilon}(\bar{\rho})\right) \bar{\rho}, \quad \mathcal{L}^{(\mathrm{int})}=c^{-1} j^{\nu} a_{\nu}, \\
\mathcal{L}^{(\mathrm{F})}=-\frac{1}{4 \mu c} f^{\nu \lambda} f_{\nu \lambda}, \quad f_{\mu \nu}=\partial_{\mu} a_{\nu}-\partial_{\nu} a_{\mu}, \\
a_{\mu}=\left(a_{0}, a_{1}, a_{2}, a_{3}\right)=(-\phi / c, \boldsymbol{a}), \\
\mathrm{D}_{t} \boldsymbol{v}+\rho^{-1} \nabla p \quad=\boldsymbol{f}_{a}, \\
\partial_{\nu} j^{\nu}=\partial_{t} \rho+\nabla \cdot \boldsymbol{j}=0, \quad j^{\nu}=(\rho c, \boldsymbol{j}), \quad \boldsymbol{j}=\rho \boldsymbol{v}, \\
\boldsymbol{f}_{a}=\boldsymbol{e}+\boldsymbol{v} \times \boldsymbol{b} \\
=\nabla \times \boldsymbol{a}, \quad \boldsymbol{e}=-\partial_{t} \boldsymbol{a}-\nabla \phi . \\
\nabla \cdot \boldsymbol{b}=0, \quad \partial_{t} \boldsymbol{b}+\nabla \times \boldsymbol{e}=0, \\
\nabla \cdot(\varepsilon \boldsymbol{e})=\rho, \quad-\partial_{t}(\varepsilon \boldsymbol{e})+\mu^{-1} \nabla \times \boldsymbol{b}=\boldsymbol{j} .
\end{gathered}
$$

where $c$ is the light velocity and $a_{\mu}$ the background field. Two parameters $\mu$ and $\varepsilon=1 /\left(c^{2} \mu\right)$ are field constants.

According to the scenario of Utiyama (1956), to begin with, we have to check the global invariance of the system under consideration. Namely, with respect to the global transformation $a_{\mu} \rightarrow a_{\mu}+\epsilon_{\mu}$ for constants $\epsilon_{\mu}$ independent of coordinates $x^{\nu}$, we ask whether the action integral $S^{\text {(total) }}$ and the governing equation (3.5) derived from it are invariant or not.

In regard to the modified Euler equation (3.5), the field $a_{\mu}$ is included only in the additional term $\boldsymbol{f}_{a}$ which includes all the components $a_{\mu}$ linearly and in derivative forms, as seen from (3.7) and (3.8). Hence, constant variation $\epsilon_{\mu}$ of $a_{\mu}$ does not give any effect on the equation (3.5). Not only the equation of motion (3.5), but the action $S^{\text {(total) }}$ of (3.1) must be invariant. Since the third Lagrangian $\mathcal{L}^{(\mathrm{F})}$ of (3.3) includes only derivative forms of $a_{\mu}$, the above global transformation causes no variation. However, regarding the second interaction Lagrangian $\mathcal{L}^{(\mathrm{int})}$, its integrand $\mathcal{J}^{\text {(int) }}$ of $S^{\text {(int) }}$ associated with the part $\mathrm{d} m=\rho \mathrm{d} \mathcal{V}$ is given by

$$
\mathcal{J}^{\text {(int) }} \equiv \mathcal{L}^{(\text {int })} \mathrm{d} \mathcal{V} c \mathrm{~d} t=c^{-1}(\rho \mathrm{d} \mathcal{V}) a_{\mu}\left(\mathrm{d} X^{\mu} / \mathrm{d} t\right) c \mathrm{~d} t=(\mathrm{d} m) a_{\mu} \mathrm{d} X^{\mu} .
$$

With respect to the global transformation $a_{\mu} \rightarrow a_{\mu}+\epsilon_{\mu}$, the action variation is

$$
\mathrm{d} S^{\mathrm{int}}=\int \mathcal{J}^{(\mathrm{int})}=(\mathrm{d} m) \epsilon_{\mu} \int_{a}^{b} \mathrm{~d} X^{\mu}=(\mathrm{d} m) \epsilon_{\mu}\left[X^{\mu}\right]_{a}^{b},
$$

for a fixed mass element $\mathrm{d} m$ of a fluid particle. It is seen that the variation $\mathrm{d} S^{\text {int }}$ does not depend on internal values of $X^{\mu}$, but depends only on its boundary values. Hence 
the constant variation $\epsilon_{\mu}$ does not give any mechanical effect on the system. Thus, the present system described by $S^{(\text {total })}$ is globally invariant for the uniform $a_{\mu}$-variation.

Next, even if the global invariance of the system $S^{\text {(total) }}$ is satisfied, one may consider local transformation by $\delta a_{\mu}\left(x^{\nu}\right)$ varying with space-time coordinates $x^{\nu}$. By substituting the transformed variable $a_{\mu}+\delta a_{\mu}\left(x^{\nu}\right)$ into $a_{\mu}$ of the action $S^{\text {(total) }}$ of $(2.28)$, the system of field equations (2.30) and (2.32) have been deduced by the action principle in section II f) In other words, Invariant Variation of the total action $S^{(\text {total })}$ of $(3.1)$ yields the system of field equations (3.5) and (3.10). The two equations of (3.9) are immediately derived from the identity $\mathrm{d}^{2} \mathcal{A} \equiv 0$ satisfied by the one-form $\mathcal{A}$, which is given in section III c)ii.

The present system is a genuine mechanical system described by the action (3.1) and the equation of motion (3.5) derived by the action principle. Then, in this case, there must be a certain background field $a_{\mu}\left(x^{\nu}\right)$ that is interacting with the flow field $v^{\mu}\left(x^{\nu}\right)$, and the interaction force is the fluid Lorentz force $\boldsymbol{f}_{a}$ of (3.7). Concerning the last point, more detailed account is given at the second half of the last section of Section IV (Summary and discussions). The background field $a_{\mu}\left(x^{\nu}\right)$ ensures the current conservation $\partial_{\nu} j^{\nu}=0$ of (3.6), which is verified in section II f) ii. based on the gauge invariance. Hence, our riddle mentioned in section I c) is resolved.

Thus, the above fluid gauge theory is proposed, according to the gauge principle of Utiyama (1956). The field of fluid-flow is required to be improved or reformed as follows. The flow field of Eulerian system is characterized by the isotropic pressure stress field. The stress field is extended to general anisotropic stress field in the flow of a perfect fluid if its motion is time-dependent and rotational. This is required by the constraint of the current conservation driven by the background gauge fields.

\section{c) Remarks on the fluid gauge theory}

Whole structure of the present theory is founded on the Gauge Principle which worked successfully in theoretical physics, particularly in the particles physics. The analyses presented so far in the present paper verifies that the scenario of the Gauge Principle works successfully in the flow field of a perfect fluid too. Here in this section some remarks on the theory are presented in order to supplement insufficient parts of the above presentation.

\section{i. Euler's equation $\mathbf{D}_{t} \boldsymbol{v}+\rho^{-1} \nabla p=0$ is still valid}

Euler's equation of motion is still valid as a family member. There is a special class of background field $\tilde{a}_{\nu}$, for which the equation (3.5) reduces to the Euler's equation of motion (2.2) for $\tilde{a}_{\nu}=\partial_{\nu} \Psi$, because, with this field, the fluid Maxwell fields $\boldsymbol{e}$ and $\boldsymbol{b}$ vanish by (3.8) (where $a_{k} \rightarrow+\partial_{k} \Psi$ and $\phi \rightarrow-\partial_{t} \Psi$ since $a_{\mu}=(-\phi / c, \boldsymbol{a}$ ) and $\partial_{0}=c^{-1} \partial_{t}$ ), and hence $\boldsymbol{f}_{a}$ of (3.7) vanishes as well.

Namely, the Euler's equation of motion (2.2), $\mathrm{D}_{t} \boldsymbol{v}+\rho^{-1} \nabla p=0$, is valid for the background field $\tilde{a}_{\nu}=\partial_{\nu} \Psi$ and the continuity equation $\partial_{t} \rho+\nabla \cdot(\rho \boldsymbol{v})=0$ is deduced from the action principle as shown in section II e) ii. Accordingly, one may say that the field $\tilde{a}_{\nu}$ itself exists, but does not show in the observable world. Hence, the rotational flow solution (Kambe 2013) mentioned insection I d) and Appendix C is yet valid as a general solution to the Euler's equation of motion $\mathrm{D}_{t} \boldsymbol{v}+\rho^{-1} \nabla p=-\nabla \Phi_{E}$, where the term $-\nabla \Phi_{E}$ on the right hand side (due to the gravitational potential $\Phi_{E}$ ) does not cause any problem in the present context. Anyway, if $\Phi_{E}$ is set to a constant, this equation reduces to (2.2). The class of flow fields governed by the Euler's equation of motion, $\mathrm{D}_{t} \boldsymbol{v}+\rho^{-1} \nabla p=0$, may be called a ground flow-state.

ii. Significance of the new force $\boldsymbol{f}_{a}$ represented with anisotropic stress tensor a. Background field: Fluid Maxwell fields

In section II e) i, we have defined the 4-potential $a_{\nu}$ by $\left(a_{0}, \boldsymbol{a}\right)$ with $a_{0}=-\phi / c$ and $\boldsymbol{a}=\left(a_{1}, a_{2}, a_{3}\right)$ and the one form $\mathcal{A}=a_{\nu} \mathrm{d} x^{\nu}$ with $x^{0} \equiv$ ct. Taking exterior differential, the field strength two-form $\mathcal{F}$ is given by 


$$
\begin{gathered}
\mathcal{F}=\mathrm{d} \mathcal{A}=\sum_{\nu<\lambda} f_{\nu \lambda} \mathrm{d} x^{\nu} \wedge \mathrm{d} x^{\lambda}, \\
f_{\nu \lambda}=\partial_{\nu} a_{\lambda}-\partial_{\lambda} a_{\nu}=-f_{\lambda \nu} .
\end{gathered}
$$

Then, a pair of fluid Maxwell fields $\boldsymbol{e}$ and $\boldsymbol{b}$ are defined:

$$
\boldsymbol{e} \equiv-\partial_{t} \boldsymbol{a}-\nabla \phi, \quad \boldsymbol{b} \equiv \nabla \times \boldsymbol{a}, \quad \text { and } \quad \overline{\boldsymbol{e}} \equiv \boldsymbol{e} / c .
$$

For the tensor $f_{\nu \lambda}$, the diagonal elements are all zero, and the element $f_{01}$ is given by $\partial_{0} a_{1}-\partial_{1} a_{0}=\left(\partial_{t} a_{1}+\partial_{1} \phi\right) / c=-e_{1} / c=-\bar{e}_{1}$ and the element $f_{12}$ given by $\partial_{1} a_{2}-\partial_{2} a_{1}=(\nabla \times \boldsymbol{a})_{3}=b_{3}$. The field strength tensor of covariant (downstairs) indices $f_{\mu \nu}$ and that of contravariant (upstairs) form $f^{\mu \nu}=\eta^{\mu \alpha} F_{\alpha \beta} \eta^{\beta \nu}$ are given by matrix forms as follows: $\ddagger$

$$
\left(f_{\nu \lambda}\right)=\left(\begin{array}{cccc}
0 & -\bar{e}_{1} & -\bar{e}_{2} & -\bar{e}_{3} \\
\bar{e}_{1} & 0 & b_{3} & -b_{2} \\
\bar{e}_{2} & -b_{3} & 0 & b_{1} \\
\bar{e}_{3} & b_{2} & -b_{1} & 0
\end{array}\right), \quad\left(f^{\nu \lambda}\right)=\left(\begin{array}{cccc}
0 & \bar{e}_{1} & \bar{e}_{2} & \bar{e}_{3} \\
-\bar{e}_{1} & 0 & b_{3} & -b_{2} \\
-\bar{e}_{2} & -b_{3} & 0 & b_{1} \\
-\bar{e}_{3} & b_{2} & -b_{1} & 0
\end{array}\right)
$$

Taking non-relativistic limit of the definition of $v^{\nu}=c u^{\nu}$ of (A.3), we have $v^{\nu}=(c, \boldsymbol{v})$. Then, from (3.15) with noting the index of $f_{k \nu}$, the equation (2.30), equivalently (2.11), can be represented in 3 -vector form as follows:

$$
\begin{gathered}
\mathrm{D}_{t} \boldsymbol{v}+\rho^{-1} \nabla p-\boldsymbol{f}_{a}=0, \\
\boldsymbol{f}_{a} \equiv \boldsymbol{e}+\boldsymbol{v} \times \boldsymbol{b}=-\partial_{t} \boldsymbol{a}-\nabla \phi+\boldsymbol{v} \times(\nabla \times \boldsymbol{a}) .
\end{gathered}
$$

The equation (3.16) is the modified Euler's equation of motion of a perfect fluid with additional force term $\boldsymbol{f}_{a}$ depending on the assumed background gauge-potential $a_{\mu}$. Note that the background potential $a_{\mu}=(-\phi / c, \boldsymbol{a})$ is analogous to the electromagnetic gauge potential $A_{\mu}$ of eq.(2.1) of Kambe (2021a), where $\Phi$ corresponds to $\phi / c$.

Using the definition (3.14) of the fields $\boldsymbol{e}$ and $\boldsymbol{b}$ and the matrix representation (3.15) of the field strength tensor $f^{\nu \lambda}$, the equation (2.32) represents the followings:

$$
\varepsilon \nabla \cdot \boldsymbol{e}=\rho, \quad-\varepsilon \partial_{t} \boldsymbol{e}+\mu^{-1} \nabla \times \boldsymbol{b}=\boldsymbol{j} .
$$

where $\varepsilon=1 /\left(c^{2} \mu\right)$. Another pair of fluid Maxwell equations is given by (3.20),

b. Second pair of fluid Maxwell equations for the background field $a_{\nu}=(-\phi / c, \boldsymbol{a})$

Using the field tensor $\left(f_{\nu \lambda}\right)$ defined by (3.13), one can derive fluid Maxwell equations of source-free type. In fact, taking one more exterior differential of $\mathcal{F}$ of (3.12), we obtain

$$
\mathrm{d} \mathcal{F}=\mathrm{d}^{2} \mathcal{A}=\sum_{\alpha<\beta<\gamma}\left(\partial_{\alpha} f_{\beta \gamma}+\partial_{\beta} f_{\gamma \alpha}+\partial_{\gamma} f_{\alpha \beta}\right) \mathrm{d} x^{\alpha} \wedge \mathrm{d} x^{\beta} \wedge \mathrm{d} x^{\gamma}=0,
$$

because $\mathrm{d}^{2} \mathcal{A} \equiv 0$. This leads to the equation, $\partial_{\alpha} f_{\beta \gamma}+\partial_{\beta} f_{\gamma \alpha}+\partial_{\gamma} f_{\alpha \beta}=0$, yielding the following pair of fluid Maxwell equations for $\boldsymbol{b}$ and $\boldsymbol{e}$ of (3.14):

$$
\nabla \cdot \boldsymbol{b}=0, \quad \partial_{t} \boldsymbol{b}+\nabla \times \boldsymbol{e}=0,
$$

where the first is obtained with $(\alpha, \beta, \gamma)=(1,2,3)$, while the second is obtained when one of $\alpha, \beta$ and $\gamma$ takes the suffix number 0 .

$\ddagger$ The latter matrix $f^{\nu \lambda}$ is to be used later to derive field equations. Practically, the matrix $f^{\nu \lambda}$ is obtained from $f_{\nu \lambda}$ with simply replacing $\overline{\boldsymbol{e}}$ by $-\overline{\boldsymbol{e}}$. 
c. Significance of $\boldsymbol{f}_{a}$ in the representation with anisotropic stress tensor

Because the formulation described so far is based on logical reasonings both physically and mathematically, the present theory is valid solidly. The present fluid gauge theory for a perfect fluid represents a broader class of flow fields than the current Eulerian field, although the Eulerian field too is valid, as stated in the part i. of this section. The current Eulerian flow field is governed by the Euler's equation of motion: $\mathrm{D}_{t} \boldsymbol{v}+\rho^{-1} \nabla p=0$. However, the present gauge theory extends the current Eulerian flow field to a wider class, covering a broader family of flow fields of a perfect fluid (an inviscid fluid).

In the presence of background field $a^{\nu}$, the governing equations are given by (3.5), (3.7) and (3.8):

$$
\begin{gathered}
\rho \mathrm{D}_{t} \boldsymbol{v}=-\nabla p+\rho \boldsymbol{f}_{a} . \\
\boldsymbol{f}_{a}=\boldsymbol{e}+\boldsymbol{v} \times \boldsymbol{b}=-\nabla \phi-\partial_{t} \boldsymbol{a}+\boldsymbol{v} \times(\nabla \times \boldsymbol{a}) .
\end{gathered}
$$

At first sight, it is surprising to see the Lorentz-type force $\boldsymbol{f}_{a}$ (acceleration correctly) in fluid-flow field which is electrically neutral. The role of charge density in the electromagnetism is played by the mass density $\rho$. Significance of the fluid Lorentz acceleration $\boldsymbol{f}_{a}$ is interpreted from the following two aspects.

Firstly, the acceleration $\boldsymbol{f}_{a}$ is independent of the mass density $\rho$ as obviously seen in (3.7), but depends on the velocity $\boldsymbol{v}$ unlike the gravity acceleration, in addition depending on the time derivative term $\partial_{t} \boldsymbol{a}$ and rotational term $\nabla \times \boldsymbol{a}$. In other words, the acceleration term $\boldsymbol{f}_{a}$ would become significant in turbulent flow fields in which flow fields are time-dependent and rotational.

It is emphasized that the fluid Lorentz acceleration $\boldsymbol{f}_{a}$ is considered to be a generalization of the pressure force $-\nabla p$. In fact, citing the equation (2.13) again:

$$
\rho \mathrm{D}_{t} v^{k}=-\partial_{j}\left(p \delta_{j k}\right)+\rho f_{k \nu} v^{\nu}, \quad(k=1,2,3 ; \quad \nu=0,1,2,3),
$$

this is equivalent to (3.21), but represented in component form.

Secondly, physical meaning of $\boldsymbol{f}_{a}$ may be given as follows. It is remarkable to find that the force field $\boldsymbol{F}_{a} \equiv \rho \boldsymbol{f}_{a}$ can be represented by the stress field $M^{\nu k}$ (where $\boldsymbol{F}_{a}$ may be called the fluid Lorentz force). In fact, for spatial components $(i, k=1,2,3)$, the $k$-th component of the force $\boldsymbol{F}_{a} \equiv \rho \boldsymbol{f}_{a}$ can be rewritten as follows:

$$
\begin{gathered}
\left(\boldsymbol{F}_{a}\right)_{k}=(\rho \boldsymbol{e}+\rho \boldsymbol{v} \times \boldsymbol{b})_{k}=-\partial_{\nu} M^{\nu k}, \quad \partial_{\nu}=\left(c^{-1} \partial_{t}, \partial_{k}\right), \\
M^{0 k}=c \epsilon(\boldsymbol{e} \times \boldsymbol{b})_{k}, \quad w_{e} \equiv \frac{1}{2} \epsilon|\boldsymbol{e}|^{2}+\frac{1}{2} \mu^{-1}|\boldsymbol{b}|^{2}=M^{00}, \\
M^{i k}=-\epsilon e_{i} e_{k}-\mu^{-1} b_{i} b_{k}+w_{e} \delta_{i k},
\end{gathered}
$$

$\left[M^{\alpha \beta} \equiv \Theta_{\mathrm{w}}^{\alpha \beta}\right.$ with $\Theta_{\mathrm{w}}^{\alpha \beta}$ defined by Eq.(33) of Kambe (2017)], where $\mu$ and $\epsilon=1 /\left(\mu c^{2}\right)$ are parameters of flow fields, and the equality $(\rho \boldsymbol{e}+\rho \boldsymbol{v} \times \boldsymbol{b})_{k}=-\partial_{\nu} M^{\nu k}$ can be shown by using (3.9) and (3.10).

The stress tensor $M^{i k}$ of (3.25) as well as the parameters $\epsilon$ and $\mu$ are analogous to those (Maxwell stress) of electromagnetism. The term $(-\nabla p)_{k}$ on the right-hand side of $(3.21)$ can be written as $-\partial_{\nu}\left(p \delta^{\nu k}\right)$, a force from the isotropic pressure stress $-p \delta^{\nu k}$.

According to the present fluid gauge theory, the state of isotropic pressure stress $p \delta^{\nu k}$ of Eulerian system is extended to the state of combined anisotropic stress $p \delta^{\nu k}+M^{\nu k}$. Namely the isotropic pressure stress $p \delta^{\nu k}$ valid at the Eulerian system is modified and augmented by an anisotropic stress $M^{\nu k}$ depending on the velocity $v^{k}$ and the time change $\partial_{t} a^{k}$, to ensure the current conservation.

The flow field described by the Euler's equation $\rho \mathrm{D}_{t} \boldsymbol{v}+\nabla p=0$ may be called a ground flow-state. Then, another flow states governed by (3.21) may be an excited flow-state. The terms "ground" and "excited" are used in analogy with quantum states, although the states here are not discrete. 


\section{IV. $\quad$ Summary And Discussions}

Theory of fluid mechanics is extended to cover time-dependent rotational flows under anisotropic stress field of a compressible perfect fluid, including turbulent flows. The Eulerian fluid mechanics is characterized with isotropic pressure stress fields. According to the general gauge principle, the current theoretical structure of fluid mechanics can be extended to a wider class of flow fields of a perfect fluid under anisotropic stress field by the Fluid Gauge Theory presented in the present paper.

Motivation of the present study is based on three observations. First one is the experimental evidence of observation of large-scale structures coexisting with turbulent flow fields; second one is a physical query of what symmetry implies the current conservation law; and third motivation is posed by a mathematical representation of the field of fluid flow, described in section I d) (Introduction).

The third one is based on the general representation of rotational flows of an ideal compressible fluid satisfying the Euler's equation presented by Kambe (2013), in which "four independent fields are newly introduced in the general solution to the Euler's equation of motion. Those fields must be either given externally or to be determined internally within the framework of theory. Present study has taken the latter approach on the understanding that the general solution predicts existence of new fields. Importantly, the very fact that the four independent fields exist in the 4dimesional linked spacetime encourages a gauge-theoretic formulation on the basis of differential forms or one-form $\mathcal{A}$ existing in the liked $4 d$-spacetime.

The last point is essential in the present study in the sense that even the fluid mechanics from the Euler's point of view has a glimpse of structures of linked $4 d$ spacetime. As an obvious example, this is seen in the equation of current conservation, $\partial j^{\nu} / \partial x^{\nu}$, represented in terms of the current 4-vector $j^{\nu}=\left(\rho c, \rho v^{k}\right)$ and the 4differential operator $\partial / \partial x^{\nu}=\left(c^{-1} \partial_{t}, \partial_{k}\right)$. We have $\partial j^{\nu} / \partial x^{\nu}=\partial_{t} \rho+\partial_{k}\left(\rho v^{k}\right)$. It is remarkable that the scalar product $\partial_{\nu} j^{\nu}$ is invariant by the Lorentz transformation.

In the $4 \mathrm{~d}$-spacetime $x^{\nu}$, by introducing a set of four fields $a_{\mu}\left(x^{\nu}\right)$, a one-form structure is defined by $\mathcal{A} \equiv a_{\mu} \mathrm{d} x^{\mu}=\rho^{-1} j^{\mu} a_{\mu} \mathrm{d} t$. Using it, an interaction action $S^{(\text {int })}=c^{-1} \int j^{\mu} a_{\mu} \mathrm{d}^{4} x^{\nu}$ was defined for the combined field of a 4-current field $j^{\mu}=\rho\left(\mathrm{d} x^{\mu} / \mathrm{d} t\right)$ and a background 4-field $a_{\mu}$. Correspondingly, a combined action $S_{c}=S^{(\mathrm{pf})}+S^{(\mathrm{int})}$ is defined by incorporating the known action of a perfect fluid $S^{(\mathrm{pf})}$, both expressed relativistically. The action principle applied to $S_{c}$ yields the equation of motion (2.11):

$$
\mathrm{D}_{t} v^{k}+\rho^{-1} \partial_{k} p=f_{k \nu} v^{\nu} . \quad f_{\mu \nu} \equiv \partial_{\mu} a_{\nu}-\partial_{\nu} a_{\mu}
$$

$\left(v^{k}=\eta^{k l} v_{l}=v_{k}\right)$. The term $f_{k \nu} v^{\nu}$ on the right-hand side is a new term (owing to interaction) added to the Euler's equation of motion given on the left-hand side. However, if the field $a_{\nu}$ takes a particular form $\tilde{a}_{\nu}=\partial_{\nu} \Psi$, then the field tensor $f_{\mu \nu}$ vanishes identically since $f_{\mu \nu}=\partial_{\mu} \partial_{\nu} \Psi-\partial_{\nu} \partial_{\mu} \Psi=0$. Then the equation (4.1) reduces to the original Euler's equation, and in addition the equation of current conservation (2.21), $\partial_{\nu} j^{\nu}=0$, is deduced for this case $(\S 2.1)$.

According to Utiyama $(1956,1987)$, the general gauge principle states as follows. "If both of the composite action $S_{c}$ and the equation of motion (4.1) are invariant under a global transformation of $\tilde{a}_{\mu}$ defined by $\tilde{a}_{\mu} \rightarrow \tilde{a}_{\mu}+\delta a_{\mu}$ for a uniform value of $\delta a_{\mu}=\epsilon_{\mu}$ (constant), then the system is said invariant globally for the $a_{\mu}$-transformation."

The constant variation $\epsilon_{\mu}$ of $\tilde{a}_{\mu}$ does not give any effect on the equation (4.1). Invariance of the action $S^{(\mathrm{int})}$ (therefore invariance of $S_{c}$ ) is also verified in $\S 3.2 . \dagger$ Hence,

$\dagger$ In $\S 3.2$, the transformation was $\tilde{a}_{\mu} \rightarrow a_{\mu}=\tilde{a}_{\mu}+\epsilon_{\mu}$. However, as far as the variation part of the action $S^{(\mathrm{int})}$ is concerned, there is no difference from that of the transformation $a_{\mu} \rightarrow a_{\mu}^{\prime}=a_{\mu}+\epsilon_{\mu}$. 
the constant variation $\epsilon_{\mu}$ does not give any mechanical effect on the system, and the system is globally invariant for the uniform $a_{\mu}$-variation.

The gauge principle reads furthermore, "Even if the global invariance of $S_{c}$ is satisfied, one may consider local transformation of $\tilde{a}_{\mu} \rightarrow a_{\mu}\left(x^{\nu}\right)=\tilde{a}_{\mu}+\delta a_{\mu}\left(x^{\nu}\right)$ with $\delta a_{\mu}\left(x^{\nu}\right)$ varying with the space-time coordinates $x^{\nu}$." The problem is now reduced to whether one can construct a physical system which is invariant under such a local gauge transformation of $a_{\mu}$ field.

The last point is interpreted as follows. There may exist a background field $a_{\mu}$ in the flow field $j^{\mu}=\rho v^{\mu}$, which interacts with the flow by the force $f_{k \nu} v^{\nu}$ (which vanished in the test of global transformation for $\tilde{a}_{\mu}$ ). Existence of a background field $a_{\mu}$ causes drastic change of our battle field. Not only the term $f_{k \nu} v^{\nu}$ is non-vanishing, but also the equations governing the new field $a_{\mu}$ should be given a physically reasonable form.

This is done by introducing the third action $S^{(\mathrm{F})}$ with the total action given by $S^{(\text {total })}=S_{c}+S^{(\mathrm{F})}$. The system is still free from external forcing. The Appendix C.2 (c) investigates the dynamics: "Free dynamical systems and the action principle of invariant variations" based on the invariant variations in the presence of background gauge field ensuring mass conservation. The background gauge field is an agent to make the dynamical system curved. This is carried out in the present formulation by implementing the connection term (i.e. the background gauge field) in the covariant derivative to make up the structure of fluid gauge theory. Finally, the free motion of physical system is described by curved geodesics. This scenario of investigating physical systems of "Free motions described by curved geodesics" is presented in Appendix C in details. In this way, the geodesic equation governing our physical system in curved motion is given by the equation (C.23) of Appendix C.2 (c), which reduces finally to the modified Euler equation (2.11) obtained in Section II c) or (3.16) of Section III c) ii.

This is the scenario of Gauge Principle. Thus, the local invariance is established, and the gauge-potential $a_{\mu}$ is taken into the system which represents a new interaction force, and a new force field $\boldsymbol{f}_{a}\left(x^{\nu}\right)$ has been introduced into our physical system by the gauge principle. Significance of the fluid Lorentz acceleration $\left(\boldsymbol{f}_{a}\right)_{k}=f_{k \nu} v^{\nu}$ is given in Section III c) ii. The force field $f_{k \nu} v^{\nu}$ is considered to be a generalization of the pressure acceleration $-\rho^{-1} \partial_{i}\left(p \delta_{i k}\right)$, as follows.

In the ground flow-state where $a_{\nu}=\partial_{\nu} \Psi$, the Euler's equation of motion (2.2) is valid. The equation can be rewritten in the form, $\rho \mathrm{D}_{t} v_{k}=\partial_{i} \sigma_{i k}\left(\equiv F_{k}\right.$ ), where the stress field $\sigma_{i k}$ is isotropic: $\sigma_{i k}$ [iso] $\equiv-p\left(x^{\nu}\right) \delta_{i k}$. One can say that the background field $\tilde{a}_{\nu} \equiv \partial_{\nu} \Psi$ itself exists, but it does not show in the observable world.

However, transition of the stress field can occur from the isotropic state $\sigma_{i k}$ [iso] to states of anisotropic stress $\sigma_{i k}[$ aniso] when the flow field (velocity field) becomes nonuniform and time-dependent. In other words, in addition to the isotropic pressure stress $\sigma_{i k}[$ iso $]=-p \delta_{i k}$ valid at the rest frame $v^{k}=0$, an anisotropic stress field $\sigma_{i k}$ [aniso] begins to grow, which depends on the velocity $v^{k}$ and the time change $\partial_{t} a_{k}$, to ensure the current conservation. To be more precise, using the fluid Maxwell stress $M^{\nu k}$ of (3.25), the force $F_{k}$ from the anisotropic stress is given by (3.24), as follows:

$$
F_{k}[\text { aniso }]=-\partial_{\nu} M^{\nu k}=-\rho \partial_{t} a_{k}-\rho \partial_{k} \phi+\rho(\boldsymbol{v} \times \boldsymbol{b})_{k} .
$$

Thus, outcomes of the Fluid Gauge Theory are summarized in this concluding section.

Based on the present fluid gauge theory, a test study has been carried out recently by M. Hashiguchi (Former iCFD researcher, Tokyo) with solving numerically the new system, finding an encouraging result on the problem: "Dust striations observed in the resonance-tube experiment' by August Kundt (1866). Brief result is shown in the presentation of Kambe (2021b). In this system there exist two different length scales with their ratio more than fifty, consistent with the photo observation of Kundt. The 
larger scale corresponds to the wavelength of the resonant acoustic wave, while the smaller one corresponds to an eddy structure generated by the background gauge field.

\section{ACKNOWLEDGMENTS}

The author expresses his deep thanks to late Professor Ryoyu Utiyama, the author of the book in Japanese "一般ゲージ場論序説 (Ippan Gauge ba ron josetsu) Introduction to the general gauge field theory". The present study aims to apply the theory to fluid systems according to the scenario of the theory of general gauge fields of Utiyama (1956, 1987). One of the motivations of the present study is that Fluid Mechanics of a perfect fluid can join in the circles of the physical theory. Also, the present author benefitted from informal discussions with Professor Yasuhide Fukumoto (Kyushu University) on the theory of general gauge fields of Utiyama.

\section{APPENDICES}

Appendix A. Some basics of linked 4d-spacetime in relativity theory

Here, some basics of the relativity theory are presented for expressions of linked 4dimensional space-time.

Suppose that a material particle or fluid particles are moving with high velocities in an inertial frame $K:\left(x^{0}, x^{1}, x^{2}, x^{3}\right)$ with $x^{0}=c t$ and $c$ the light velocity. In a time interval $\mathrm{d} t$, the position of the particle changes with time and its displacement is given by a 4 -vector:

$$
\mathrm{d} x^{\mu}=\left(c \mathrm{~d} t, \mathrm{~d} X^{1}, \mathrm{~d} X^{2}, \mathrm{~d} X^{3}\right), \quad \mathrm{d} X^{k}=v^{k} \mathrm{~d} t \quad(k=1,2,3),
$$

where $\mu=0,1,2,3$, and the upper-case notation $\mathrm{d} X^{k}$ denotes displacement of a material (fluid) particle with $v^{k}$ components of 3 -velocity $\boldsymbol{v}$. In the relativity theory, an infinitesimal interval $\mathrm{d} s$ is defined by its squared form, $\mathrm{d} s^{2}=\mathrm{d} x_{\mu} \mathrm{d} x^{\mu}$, which is a scalar product of a line-element 4 -vector $\mathrm{d} x^{\mu}$ with its covariant version $\mathrm{d} x_{\mu}=$ $\eta_{\mu \nu} \mathrm{d} x^{\nu}=\left(-c \mathrm{~d} t, \mathrm{~d} X^{1}, \mathrm{~d} X^{2}, \mathrm{~d} X^{3}\right)$, where $\eta_{\mu \nu}$ is the Minkowski metric, sometimes called the Lorentz metric, defined by $\eta_{\mu \nu}=\eta^{\mu \nu}=\operatorname{diag}(-1,1,1,1)$. Hence, we have $\mathrm{d} s^{2}=\mathrm{d} x_{\mu} \mathrm{d} x^{\mu}=\eta_{\mu \nu} \mathrm{d} x^{\mu} \mathrm{d} x^{\nu}=-c^{2} \mathrm{~d} t^{2}+|\mathrm{d} \boldsymbol{X}|^{2}$. The interval $\mathrm{d} s$ is a relativistic invariant, i.e. invariant under the Lorentz transformation (see Appendix B of Kambe (2021a)).

Another relativistic invariant is the proper time $\tau$. Its increment $\mathrm{d} \tau$ is defined by the time increment (multiplied by $c$ ) in the instantaneously rest frame where $\boldsymbol{v}=0$. Squared interval of the proper time is defined by $\mathrm{d} \tau^{2}=-\mathrm{d} x_{\nu} \mathrm{d} x^{\nu}=-\mathrm{d} s^{2}$. From this, noting $\mathrm{d} X^{k}=v^{k} \mathrm{~d} t$, we obtain

$$
\mathrm{d} \tau=c \mathrm{~d} t \sqrt{1-\beta^{2}}, \quad \beta \equiv v / c, \quad v=\sqrt{v_{k} v^{k}}
$$

Using the displacement $\mathrm{d} X^{\nu}$ of a fluid particle $P$, its relativistic 4-velocity is defined by

$$
u^{\nu}=\frac{\mathrm{d} X^{\nu}}{\mathrm{d} \tau}=\left(\frac{1}{\sqrt{1-\beta^{2}}}, \frac{\boldsymbol{v}}{c \sqrt{1-\beta^{2}}}\right), \quad \boldsymbol{v}=\left(v^{k}\right)=\left(\mathrm{d} X^{k} / \mathrm{d} t\right) .
$$

This fluid particle $P$ is moving with the 4 -velocity $u^{\nu}$ relative to the frame $x^{\mu}$.

Appendix B. Relativistic formulation of action principle: three mechanical systems Relativistic formulation of the variational principle is presented for three fundamental mechanical systems. We consider how covariant derivatives or gauge invariant equations are deduced from the principle of least action, i.e. from the invariant variations. 


\section{Appendix B.1. Free motion of a point mass}

The action of a free material particle of rest mass $m$ is given by

$$
S^{(\mathrm{m})}=-m c \int_{a}^{b} \mathrm{~d} \tau \equiv \int \mathcal{L}^{(\mathrm{m})} \mathrm{d} t
$$

(its derivation, see Landau \& Lifshitz $(1975, \S 8)$ ), where the present $\mathrm{d} \tau$ is equivalent to the $\mathrm{d} s$ of Landau \& Lifshitz owing to the difference of the metric tensor definitions. $f$ Since $\mathrm{d} \tau=c \mathrm{~d} t \sqrt{1-\beta^{2}}$ from (A.2) of the main text, the Lagrangian $\mathcal{L}^{(\mathrm{m})}$ is

$$
\mathcal{L}^{(\mathrm{m})}=-m c^{2} \sqrt{1-\beta^{2}} .
$$

Principle of least action requires vanishing of the variation $\delta S^{(\mathrm{m})}$ :

$$
\delta S^{(\mathrm{m})}=-m c \delta \int \mathrm{d} \tau=0 .
$$

Since $\mathrm{d} \tau^{2}=-\eta_{\mu \nu} \mathrm{d} x^{\nu} \mathrm{d} x^{\nu}$ (Appendix A), we obtain $\delta\left(\mathrm{d} \tau^{2}\right)=2 \mathrm{~d} \tau \delta \mathrm{d} \tau=-2 \eta_{\mu \nu} \mathrm{d} x^{\nu} \delta \mathrm{d} x^{\nu}$. Hence, we obtain the followings:

$$
\begin{gathered}
\delta \mathrm{d} \tau=-\eta_{\mu \nu} \frac{\mathrm{d} x^{\nu}}{\mathrm{d} \tau} \delta \mathrm{d} x^{\nu}=-u_{\nu} \delta \mathrm{d} x^{\nu}=-u_{\nu} \mathrm{d}\left(\delta x^{\nu}\right) . \\
\delta S^{(\mathrm{m})}=m c \int_{a}^{b} u_{\nu} \mathrm{d}\left(\delta x^{\nu}\right)=m c\left[u_{\nu} \delta x^{\nu}\right]_{a}^{b}-m c \int_{a}^{b} \delta x^{\nu} \frac{\mathrm{d} u^{\nu}}{\mathrm{d} \tau} \mathrm{d} \tau .
\end{gathered}
$$

where the integration limits of lower $a$ and upper $b$ are added.

To get the equation of motion, different trajectories are compared by assuming that the variation $\delta x^{\nu}$ is arbitrary within the interval $[a, b]$, but vanishes at $a$ and $b$. Then, the principle of least action determines the trajectory by $\delta S^{(\mathrm{m})}=0$. Thus we obtain

$$
\nabla_{\tau} u^{\nu} \equiv \frac{\mathrm{d}}{\mathrm{d} \tau} u^{\nu}=0
$$

Namely, the 4-velocity $u^{\nu}$ of the free particle is constant in time, as well-known.

\section{Appendix B.2. Free motion of a perfect fluid}

A perfect fluid is defined as a continuum object (a continuous matter) in the $4 \mathrm{~d}-$ spacetime $x^{\mu}=(c t, \boldsymbol{x})=\left(x^{0}, x^{1}, x^{2}, x^{3}\right)$, characterized with a mass density $\rho\left(x^{\mu}\right)$ in motion with 3 -velocity $\boldsymbol{v}=\left(v^{1}, v^{2}, v^{3}\right)$ and without any internal mechanism of energy dissipation. During its motion, the entropy change $\Delta s=T^{-1}\left(\Delta \epsilon+p \Delta \mathcal{V}_{1}\right)$ is assumed to vanish, where $s$ and $\epsilon$ are thermodynamic variables of entropy and internal energy per unit mass with the volume element $\Delta \mathcal{V}_{1}$ defined by $1 / \rho$. The pressure and temperature are denoted by $p$ and $T$. Flow variables such as $\rho, p, \boldsymbol{v}$, etc. are represented by continuous differentiable functions of the coordinates $x^{\mu}=(c t, \boldsymbol{x})$.

The action for free motion of a perfect fluid is given by

$$
S^{(\mathrm{pf})}=\int\left[\int L^{(\mathrm{pf})} \bar{\rho} \overline{\mathrm{d} \mathcal{V}}\right] \mathrm{d} \tau=\int\left[\int \mathcal{L}_{(\mathrm{pf})}\left(x^{\nu}\right) \mathrm{d} \mathcal{V}\right] c \mathrm{~d} t
$$

$\ddagger$ The negative sign is added in front of the integral $\int_{a}^{b} \mathrm{~d} \tau$, because it takes its maximum value along a straight geodesic line (see the text cited). 


$$
\begin{gathered}
L^{(\mathrm{pf})}=-c^{-1}\left(m_{1} c^{2}+\overline{\epsilon(\rho)}\right)=-c\left(1+c^{-2} \overline{\epsilon(\rho)}\right) \quad \text { since } m_{1}=1, \\
\mathrm{~d} \tau=c \mathrm{~d} t \sqrt{1-\beta^{2}}, \quad \bar{\rho} \overline{\mathrm{d} \mathcal{V}}=\rho \mathrm{d} \mathcal{V}=\mathrm{d} m, \quad \bar{\rho}=\rho \sqrt{1-\beta^{2}}, \\
\mathcal{L}_{\mathrm{pf}} \mathrm{d} \mathcal{V} \equiv L^{(\mathrm{pf})} \bar{\rho} \overline{\mathrm{d} \mathcal{V}} \sqrt{1-\beta^{2}}=-c^{-1}\left(m_{1} c^{2}+\bar{\epsilon}(\bar{\rho})\right) \sqrt{1-\beta^{2}}[\rho \mathrm{d} \mathcal{V}],
\end{gathered}
$$

where the integration within the first bracket [ ] of (B.7) is done with respect to d $\mathcal{V}$ of material location $\left(\mathrm{d} X^{1} \mathrm{~d} X^{2} \mathrm{~d} X^{3}\right)$, and overlined values denote proper values (i.e. the values in the comoving frame where the fluid is at rest). The terms $\bar{\epsilon}$ and $\bar{\rho}$ denote the proper internal energy and density, with $\mathrm{d} \tau=c \mathrm{~d} t \sqrt{1-\beta^{2}}$ the proper time interval, $\bar{\rho} \overline{\mathrm{d} \mathcal{V}}$ denotes the proper mass element $\mathrm{d} m$, and $\mathrm{d} \mathcal{V}=\mathrm{d} X^{1} \mathrm{~d} X^{2} \mathrm{~d} X^{3}$ is a volume element associated with the mass $\mathrm{d} m$. The $m_{1}=1$ (unit mass) is added to clarify physical meaning of the term $\left(m_{1} c^{2}+\bar{\epsilon}(\bar{\rho})\right)$ as relativistic proper internal energy per unit mass including the rest-mass energy $m_{1} c^{2}$. (For the form of $L^{(\mathrm{pf})}$, see Kambe (2020), Dewar (1977), or Salmon (1988b).)

The Lagrangian per a volume-element $\mathrm{d} \mathcal{V}$ is given by $\mathcal{L}_{\mathrm{pf}}=-c^{-1} \sqrt{1-\beta^{2}}\left(m_{1} c^{2}+\right.$ $\epsilon(\rho)) \rho$. This is the proper Lagrangian with respect to an inertial frame. Its nonrelativistic limit (as $\beta \rightarrow 0$ ) per unit mass is given as $c^{-1}\left(-m_{1} c^{2}+\frac{1}{2} m_{1} v^{2}-\epsilon+\cdots\right)$. The first term $m_{1} c^{2}$ denotes the mass energy (with negative sign attached) and neglected in the non-relativistic limit. Subsequent two terms $\left(\frac{1}{2} m_{1} v^{2}-\epsilon\right)$ per unit mass is equivalent to the traditional non-Relativistic Lagrangian $\Lambda_{\mathrm{nR}}$ :

$$
\Lambda_{\mathrm{nR}}=\frac{1}{2} v^{2}-\epsilon, \quad S^{(\mathrm{nR})}=\int\left[\int \Lambda_{\mathrm{nR}} \rho \mathrm{d} \mathcal{V}\right] \mathrm{d} t,
$$

where the front factor $c^{-1}$ and the integration element $c \mathrm{~d} t$ make the $\mathrm{d} t$ in the above integral. Let us take variation of $S^{(\mathrm{pf})}$ of (B.7):

$$
\delta S^{(\mathrm{pf})}=\iint\left[\bar{L}^{(\mathrm{pf})} \delta \mathrm{d} \tau+\delta \bar{L}^{(\mathrm{pf})} \mathrm{d} \tau\right] \mathrm{d} m=\delta S_{1}^{(\mathrm{pf})}+\delta S_{2}^{(\mathrm{pf})}
$$

Variation is taken keeping the mass element $\mathrm{d} m=\overline{\rho \mathrm{d} \mathcal{V}}$ fixed and written as $\Delta m=\bar{\rho} \overline{\Delta \mathcal{V}}$. Then the second term is, under the thermodynamic condition $\left.\delta \bar{\epsilon}\right|_{s: \text { fixed }}=-\bar{p} \delta(1 / \bar{\rho})$,

$$
\begin{aligned}
(\Delta m) \delta \bar{L}^{(\mathrm{pf})} \mathrm{d} \tau & =-c^{-1} \Delta m \delta \overline{\epsilon(\rho)} \mathrm{d} \tau=-c^{-1} \Delta m\left((\bar{\rho})^{-1} \delta \bar{p}-\delta(\bar{p} / \bar{\rho})\right) \mathrm{d} \tau \\
& =-c^{-1} \Delta m \frac{1}{\bar{\rho}} \partial_{\nu} \bar{p} \delta x^{\nu} \mathrm{d} \tau+c^{-1} \delta\left(\bar{\rho} \overline{\Delta \mathcal{V}} \frac{\bar{p}}{\bar{\rho}}\right) \mathrm{d} \tau
\end{aligned}
$$

while for the first term, using the definition $\mathrm{d} \tau=\sqrt{-\eta_{\mu \nu} \mathrm{d} x^{\nu} \mathrm{d} x^{\nu}}$ together with (B.4),

$$
\begin{aligned}
(\Delta m) \bar{L}^{(\mathrm{pf})} \delta \mathrm{d} \tau & =c^{-1}\left(c^{2}+\bar{\epsilon}(\bar{\rho})\right) \Delta m u_{\nu} \mathrm{d}_{\tau}\left(\delta x^{\nu}\right) \mathrm{d} \tau \\
& =c^{-1}\left(c^{2}+\bar{\epsilon}(\bar{\rho})\right) \Delta m\left(-\left(\frac{\mathrm{d}}{\mathrm{d} \tau} u_{\nu}\right) \delta x^{\nu}+\frac{\mathrm{d}}{\mathrm{d} \tau}\left(u_{\nu} \delta x^{\nu}\right)\right) \mathrm{d} \tau .
\end{aligned}
$$

Thus, summing up both terms, we obtain

$$
\begin{aligned}
\Delta m\left[\bar{L}^{(\mathrm{pf})} \delta \mathrm{d} \tau\right. & \left.+\delta \bar{L}^{(\mathrm{pf})} \mathrm{d} \tau\right]=-c \Delta m\left(\frac{\mathrm{d}}{\mathrm{d} \tau} u_{\nu}+c^{-2} \frac{1}{\bar{\rho}} \partial_{\nu} \bar{p}\right) \delta x^{\nu} \mathrm{d} \tau \\
& +c^{-1} \delta(\bar{p} \overline{\Delta \mathcal{V}}) \mathrm{d} \tau+c\left(1+O\left(\beta^{2}\right)\right) \Delta m \mathrm{~d}_{\tau}\left(u_{\nu} \delta x^{\nu}\right) \mathrm{d} \tau
\end{aligned}
$$


Since the two terms of the second line (B.14) do not give any contribution to the variation by the reasons explained below, we are concerned with the first line (B.14) only for the variational analysis. For arbitrary variations $\delta x^{k}\left(k=1,2,3\right.$ with $\left.\delta x^{0}=0\right)$, vanishing of the total variations requires the following equation, to the leading order of the series with respect to the $\beta^{2}(\ll 1)$ expansion:

$$
c^{2} \frac{\mathrm{d}}{\mathrm{d} \tau} u_{k}+\frac{1}{\rho} \partial_{k} p=0,
$$

where $\mathrm{d} \tau=c \mathrm{~d} t \sqrt{1-\beta^{2}}$. We note that $\bar{\rho}=\rho \sqrt{1-\beta^{2}}$ (Kambe (2020) Appendix B.1) and $\bar{p}=p$ (Agmon 1977). Hence using $u_{k}=\left(v_{k} /\left[c \sqrt{1-\beta^{2}}\right]\right)$ from (A.3), the above becomes

$$
\frac{1}{\sqrt{1-\beta^{2}}} \frac{\mathrm{D}}{\mathrm{D} t} \frac{v_{k}}{\sqrt{1-\beta^{2}}}+\frac{1}{\rho} \partial_{k} p=0,
$$

where $(\mathrm{D} / \mathrm{D} t) \equiv \mathrm{D}_{t}=\partial_{t}+\boldsymbol{v} \cdot \nabla$ is the convective derivative defined by (2.4). Transforming this into a contravariant form by multiplying $\eta^{i k}$ (no change except the change of indices from lower to upper), the leading order form of the equation becomes

$$
\nabla_{\tau}^{(\mathrm{pf})} v^{k} \equiv \mathrm{D}_{t} v^{k}+\rho^{-1} \partial_{k} p=0
$$

since $1 / \sqrt{1-\beta^{2}}=1+O\left(\beta^{2}\right)$ and $\partial^{k}=\partial_{k}$. This is nothing but the Euler's equation of motion in the form of $(2.2)$.

The equation (C.10) is a geodesic equation of free motion of perfect fluid of a constant density $\rho_{*}$, expressed as $\widehat{\nabla}_{t} \boldsymbol{u} \equiv \partial_{t} \boldsymbol{u}+(\boldsymbol{u} \cdot \nabla) \boldsymbol{u}+\nabla\left(p / \rho_{*}\right)=0$. Here we used the symbol $\nabla_{\tau}^{(\text {pf })}$ to denote the covariant $\tau$-derivative of perfect fluid, because the leading term of (B.14) can be written as

$$
\Delta m\left[\bar{L}^{(\mathrm{pf})} \delta \mathrm{d} \tau+\delta \bar{L}^{(\mathrm{pf})}\right]=-\frac{1}{c \sqrt{1-\beta^{2}}} \Delta m\left(\nabla_{\tau}^{(\mathrm{pf})} v^{k}\right) \mathrm{d} \tau \delta x^{k}=0
$$

with the second line of (B.14) deleted.

In regard to the second line of (B.14), the factor $\mathrm{d}_{\tau}\left(u_{\nu} \delta x^{\nu}\right) \mathrm{d} \tau$ of the last term can be integrated with respect to $\tau$. Hence the last term does not give any contribution to the variation, while the remaining first term leads to total variation of the integration $I \equiv \int p \mathrm{~d} \mathcal{V}$ in the rest frame, which is fixed for the variation, i.e. $\delta I=0$. In fact, the kinetic theory of statistical mechanics implies that $\int p \mathrm{~d} \mathcal{V}$ denotes $2 / 3$ of total kinetic energy $E$ of particles composing an ideal gas, which is invariant in free state. Thus, the second line of (B.14) does not give any contribution to the variation,

\section{Appendix B.3. Free motion of a perfect fluid under interaction action $S^{\text {int }}$}

According to the sections III a) and b) in the main text, one can define a composite action $S_{c}$ by using the action $S^{(\mathrm{pf})}$ of a perfect fluid of (B.7) and the action $S^{(\mathrm{int})}$ of (2.6) for interaction of current $j^{\nu}$ and the gauge field $a_{\nu}$. Let us define

$$
\begin{aligned}
S_{c} & \equiv S^{(\mathrm{pf})}+S^{(\mathrm{int})}, \\
S^{(\mathrm{pf})} & \equiv-c \int \rho \mathrm{d} \mathcal{V} \int\left(1+c^{-2} \bar{\epsilon}(\rho)\right) \mathrm{d} \tau=\int \mathcal{L}^{4} x=\mathrm{d} \mathcal{V} \mathrm{d} t_{c} \\
S^{(\mathrm{pf})} \mathrm{d}^{4} x, & \equiv \int \mathcal{L}^{(\text {int })} \mathrm{d}^{4} x, \quad \mathcal{L}^{(\text {int })} \equiv c^{-1} j^{\mu} a_{\mu}, \quad j^{\mu}=\rho v^{\mu},
\end{aligned}
$$

where $\mathcal{L}^{(\mathrm{pf})} \equiv-c \rho\left(1+c^{-2} \bar{\epsilon}\right) \sqrt{1-\beta^{2}}$, and $v^{\mu}=\mathrm{d} X^{\mu} / \mathrm{d} t$. 
To find the equations of motion, the action principle is applied to the composite action $S_{c}$, by assuming the gauge potential $a_{\nu}$ given and vary only the position coordinate $X_{p}^{k}$ of fluid particles moving with the velocity $\mathrm{D}_{t} X_{p}^{k}$ along their trajectories. On the other hand, to find the equations governing the $a_{\nu}$, we vary only the gauge-potential $a_{\nu}$ with assuming the fluid motion given and fixed. However, to carry out the latter variation, we have to define a third action to characterize the background field $a_{\nu}$ and add it to $S_{c}$, (which is postponed to the next Appendix B.4), Here, we carry out the former variation, then the action principle applied to the varied $S_{c}$ should yield the equation of fluid motion.

By the way, under the requirement of invariance of $S^{(\text {int })}$ to the gauge transformation of potential $a_{\nu}$, the current conservation law $\partial_{\nu} j^{\nu}=0$ is deduced in section II a) Hence, we assume the invariance of the mass element $\mathrm{d} m \equiv \rho \mathrm{d} \mathcal{V}$ of a fluid particle during the motion along its trajectory when variations are taken with respect to the particle position $X_{p}^{k}$.

Regarding the action $S^{(\mathrm{pf})}$ of (B.7). Its variation is given by (B.14), and its variation-integrand $\delta \mathcal{J}^{(\mathrm{pf})}$ is found as follows:

$$
\delta \mathcal{J}^{(\mathrm{pf})}=-c \Delta m\left(\frac{\mathrm{d}}{\mathrm{d} \tau} u_{\nu}+c^{-2} \frac{1}{\bar{\rho}} \partial_{\nu} \bar{p}\right) \delta x^{\nu} \mathrm{d} \tau+\text { higher order terms of } O\left(\beta^{2}\right) .
$$

Before taking variation of the interaction action $S^{(\mathrm{int})}=\iint \mathcal{J}^{(\mathrm{int})}$, we rewrite its integrand $\mathcal{J}^{\text {(int) }}$ as follows (since $\mathrm{d}^{4} x=c \mathrm{~d} t \mathrm{~d} \mathcal{V}$ ):

$$
\mathcal{J}^{(\text {int })}=(\rho \mathrm{d} \mathcal{V}) v^{\nu} a_{\nu} \mathrm{d} t=(\mathrm{d} m) \frac{\mathrm{d} X^{\nu}}{\mathrm{d} t} a_{\nu} \mathrm{d} t=(\mathrm{d} m) a_{\nu} \mathrm{d} X^{\nu},
$$

where (2.9) and $v^{\mu}=\mathrm{d} X^{\mu} / \mathrm{d} t$ are used with $\mathrm{d} m=\rho \mathrm{d} \mathcal{V}$. Its variation is given by

$$
\begin{aligned}
& \delta \mathcal{J}^{(\text {int })}=(\Delta m)\left(a_{\nu} \mathrm{d}\left(\delta x^{\nu}\right)+\delta a_{\nu} \mathrm{d} x^{\nu}\right)=\overline{\Delta m}\left(-\mathrm{d} a_{\nu} \delta x^{\nu}+\delta a_{\nu} \mathrm{d} x^{\nu}+\mathrm{d}\left(a_{\nu} \delta x^{\nu}\right)\right) \\
&=\overline{\Delta m}\left[-\frac{\partial a_{\nu}}{\partial x^{\mu}} \mathrm{d} x^{\mu} \delta x^{\nu}+\frac{\partial a_{\nu}}{\partial x^{\mu}} \delta x^{\mu} \mathrm{d} x^{\nu}+\mathrm{d}\left(a_{\nu} \delta x^{\nu}\right)\right] \\
&=\overline{\Delta m}\left(\frac{\partial a_{\mu}}{\partial x^{\nu}}-\frac{\partial a_{\nu}}{\partial x^{\mu}}\right) \frac{\mathrm{d} x^{\mu}}{\mathrm{d} \tau} \delta x^{\nu} \mathrm{d} \tau+c \overline{\Delta m} \frac{\mathrm{d}\left(a_{\nu} \delta x^{\nu}\right)}{\mathrm{d} \tau} \mathrm{d} \tau \\
&= \overline{\Delta m} f_{\nu \mu} u^{\mu} \delta x^{\nu} \mathrm{d} \tau+\overline{\Delta m} \frac{\mathrm{d}\left(a_{\nu} \delta x^{\nu}\right)}{\mathrm{d} \tau} \mathrm{d} \tau, \\
& f_{\mu \nu} \equiv \partial_{\mu} a_{\nu}-\partial_{\nu} a_{\mu}=-f_{\nu \mu} .
\end{aligned}
$$

Thus, summing up the two variations (B.22) and (B.24), we obtain

$$
\delta \mathcal{J}^{(\mathrm{fl}+\mathrm{a})} \equiv \delta \mathcal{J}^{(\mathrm{pf})}+\delta \mathcal{J}^{(\mathrm{int})}=-c^{-1} \overline{\Delta m}\left[c^{2} \frac{\mathrm{d}}{\mathrm{d} \tau} u_{\nu}+\frac{1}{\bar{\rho}} \partial_{\nu} \bar{p}-c f_{\nu \mu} u^{\mu}\right] \mathrm{d} \tau \delta x^{\nu}
$$

by neglecting higher order terms and vanishing integrals with respect to $\tau$. Requiring $\delta \mathcal{J}^{(\mathrm{fl}+\mathrm{a})}=0$ for arbitrary variation $\delta x^{\nu}$, this leads to

$$
c^{2} \frac{\mathrm{d}}{\mathrm{d} \tau} u_{\nu}+\frac{1}{\bar{\rho}} \partial_{\nu} \bar{p}-c f_{\nu \mu} u^{\mu}=0 .
$$

Remembering that the first two terms reduced to (B.16) in the Appendix B.2, we find that the above equation reduces to the following $(\nu=0,1,2,3 ; k=1,2,3)$ :

$$
\frac{1}{\sqrt{1-\beta^{2}}} \frac{\mathrm{D}}{\mathrm{D} t} \frac{v_{k}}{\sqrt{1-\beta^{2}}}+\frac{1}{\rho} \partial_{k} p-f_{k \nu} v^{\nu}=0,
$$


where $u_{k}=\left(v_{k} /\left[c \sqrt{1-\beta^{2}}\right]\right)$ is used from (A.3), Extending the reasoning of Appendix B.2, leading order form of this equation becomes

$$
\nabla_{\tau}^{(\mathrm{pfa})} v^{k} \equiv \mathrm{D}_{t} v^{k}+\rho^{-1} \partial_{k} p-f_{k \nu} v^{\nu}=0, \quad(\nu=0,1,2,3) .
$$

since $1 / \sqrt{1-\beta^{2}}=1+O\left(\beta^{2}\right)$. Thus we have found an extended equation of Euler's equation in the presence of background field $a_{\mu}$ giving rise to new third term.

\section{Appendix B.4. Free motion of a perfect fluid : in the presence of gauge field re-ensuring mass conservation}

To make the fluid system self-contained, we need a third action $S^{(\mathrm{F})}=\int \mathcal{L}^{(\mathrm{F})} \mathrm{d} \Omega$ in addition to $S^{(\mathrm{pf})}$ and $S^{(\mathrm{int})}$ of Appendix B.3, to govern free-state of the background field $a_{\nu}$, describing only on the property of the field itself. According to section II e) iv. a possible form of the free-Lagrangian $\mathcal{L}^{(\mathrm{F})}$ is proposed to be quadratic in $\partial_{\mu} a_{\nu}$ or $f_{\mu \nu}$, because the variation of $S^{(\mathrm{F})}$ reduces the degree by one with resulting equation becoming linear to $\partial_{\mu} a_{\nu}$. The only Lorentz-invariant quadratic form is a multiple of $f_{\mu \nu} f^{\mu \nu}$. This satisfies the requirement (i) of section II e) iv. namely, the fluid Maxwell fields $\boldsymbol{e}$ and $\boldsymbol{b}$ should be ensured to vanish when the background field $a_{\nu}$ takes the special form $\tilde{a}_{\nu}=\partial_{\mu} \Psi$. This means that the original Euler's equation of motion (2.2) is valid in spite of the existence of the field $a_{\nu}$.

Following the propositions of Kambe (2017, 2020), our fluid system is a combined system of two fields: a fluid-current field $\mathrm{J}^{\nu}$ and a background field $a_{\nu}$ ensuring the continuity equation. Accordingly, the Lagrangian density $\mathcal{L}$ consists of three terms: Lagrangians of $(i)$ perfect fluid $\mathcal{L}^{(\mathrm{pf})},($ ii $)$ back-ground field $\mathcal{L}^{(\mathrm{F})}$ and $($ iii $)$ their mutual interaction $\mathcal{L}^{\text {(int) }}$. Total Lagrangian is expressed as $\mathcal{L}^{(\text {total })}=\mathcal{L}^{(\mathrm{pf})}+\mathcal{L}^{(\text {int })}+\mathcal{L}^{(\mathrm{F})}$.

\section{(a) Total action}

The total action $S^{(\text {total })}$ is given by

$$
S^{(\text {total })}=\iint\left(\mathcal{L}^{(\mathrm{pf})}+\mathcal{L}^{(\mathrm{int})}+\mathcal{L}^{(\mathrm{F})}\right) c \mathrm{~d} t \mathrm{~d} \mathcal{V}
$$

where $\mathrm{d}^{4} x=\mathrm{d} \Omega=c \mathrm{~d} t \mathrm{~d} \mathcal{V}$, and $\mathrm{d} \mathcal{V}=\mathrm{d} x^{1} \mathrm{~d} x^{2} \mathrm{~d} x^{3}=\overline{\mathrm{d} \mathcal{V}} \sqrt{1-\beta^{2}}$. Since $\rho \sqrt{1-\beta^{2}}=\bar{\rho}$, the mass element $\mathrm{d} m=\rho \mathrm{d} \mathcal{V}$ is invariant, i.e. $\bar{\rho} \overline{\mathrm{d} \mathcal{V}}=\rho \mathrm{d} \mathcal{V}$.

The Lagrangian densities are defined by

$$
\mathcal{L}^{(\mathrm{pf})}=-c^{-1} \bar{\rho}\left(c^{2}+\overline{\epsilon(\rho)}\right), \quad \mathcal{L}^{(\mathrm{int})}=c^{-1} j^{\nu} a_{\nu}, \quad \mathcal{L}^{(\mathrm{F})}=-\frac{1}{4 \mu c} f^{\nu \lambda} f_{\nu \lambda},
$$

where $j^{\nu} \equiv \bar{\rho} v^{\nu}=\rho \sqrt{1-\beta^{2}} v^{\nu}=\rho \mathrm{d} X^{\nu} / \mathrm{d} t$ is the 4-current density and $v^{\nu}$ is the relativistic 4 -velocity, defined by

$$
v^{\nu}=c \frac{\mathrm{d} X^{\nu}}{\mathrm{d} \tau}=\left(\frac{c}{\sqrt{1-\beta^{2}}}, \frac{\boldsymbol{v}}{\sqrt{1-\beta^{2}}}\right) \equiv c u^{\nu}, \quad j^{\nu}=\rho \frac{\mathrm{d} X^{\nu}}{\mathrm{d} t}=\rho(c, \boldsymbol{v}) .
$$

The tensor $f_{\nu \lambda}$ is field-strength tensor of background field, defined by

$$
f_{\nu \lambda}=\partial_{\nu} a_{\lambda}-\partial_{\lambda} a_{\nu}
$$

where $a_{\nu}=\left(-\phi / c, a_{k}\right)$ is a 4 -potential of the background field. Here in the present fluid system, we use lower-case letters to denote field variables corresponding to Electromagnetic variables where upper-case letters are used in section II a) of Kambe (2021a).

To find the equations governing the background field $a_{\nu}$, we apply the principle of least action to the action $S^{\text {(total) }}$, by assuming the fluid motion given and vary the 
potential $a_{\nu}$ only. On the other hand, to find the equations of fluid motion, we assume the field potential $a_{\nu}$ given and vary only the trajectory of the fluid particle ( $\boldsymbol{X}$ or $\left.\boldsymbol{v}=\partial_{t} \boldsymbol{X}\right)$. The latter variation is equivalent to what is done in Appendix B.3. The equation (B.29) derived there is cited as the equation (B.36) at the end of this section.

We carry out the former variation, anticipating to deduce the current conservation $\partial_{\nu} j^{\nu}=0$ of fluid flows.

(b) Variation with respect to $a_{\nu}$

We have two Lagrangian densities which include the field $a_{\nu}: \mathcal{L}^{\text {(int) }}=c^{-1} v^{\nu} a_{\nu}$ and $\mathcal{L}^{(\mathrm{F})}=-\frac{1}{4 \mu c} f^{\nu \lambda} f_{\nu \lambda}$. First, we note $\delta\left(f^{\nu \lambda} f_{\nu \lambda}\right)=2 f^{\nu \lambda}\left(\delta f_{\nu \lambda}\right)$. This is because

$$
\left(\delta f^{\nu \lambda}\right) f_{\nu \lambda}=\left(\delta f^{\nu \lambda}\right) \eta_{\nu \alpha} \eta_{\lambda \beta} f^{\alpha \beta}=f^{\alpha \beta}\left(\delta f_{\alpha \beta}\right) .
$$

Therefore, variation of $\mathcal{L}^{(\mathrm{int})}+\mathcal{L}^{(\mathrm{F})}$ is given by

$$
\begin{aligned}
c \delta \mathcal{L}^{(\mathrm{int})}+c \delta \mathcal{L}^{(\mathrm{F})} & =j^{\nu} \delta a_{\nu}-\frac{1}{2 \mu} f^{\nu \lambda} \delta f_{\nu \lambda}=j^{\nu} \delta a_{\nu}-\frac{1}{2 \mu} f^{\nu \lambda} \frac{\partial}{\partial x^{\nu}} \delta a_{\lambda}+\frac{1}{2 \mu} f^{\nu \lambda} \frac{\partial}{\partial x^{\lambda}} \delta a_{\nu} \\
& =\left(j^{\nu}-\frac{1}{\mu} \frac{\partial}{\partial x^{\lambda}} f^{\nu \lambda}\right) \delta a_{\nu} .
\end{aligned}
$$

where the term $-(1 / 2 \mu) f^{\nu \lambda} \partial_{\nu}\left(\delta a_{\lambda}\right)$ next to the last on the upper line can be equated to the last term $(1 / 2 \mu) f^{\nu \lambda} \partial_{\lambda}\left(\delta a_{\nu}\right)$ by using the anti-symmetric property, $-f^{\nu \lambda}=f^{\lambda \nu}$, and interchanging the indices $\nu$ and $\lambda$, and the last two terms on the upper line are combined to give $(1 / \mu) f^{\nu \lambda} \partial_{\lambda}\left(\delta a_{\nu}\right)$, and finally carrying out integration-by-parts leads to the second line of (B.34) with omitting the term of the form $\partial_{\lambda}\left[(1 / \mu) f^{\nu \lambda} \delta a_{\nu}\right]$, which is transformed to vanishing boundary integrals in the original action integral.

Requiring vanishing of the varied Lagrangian $\delta \mathcal{L}^{(\mathrm{int})}+\delta \mathcal{L}^{(\mathrm{F})}=0$ for arbitrary variation $\delta a_{\nu}$, we obtain

$$
\frac{\partial}{\partial x^{\lambda}} f^{\nu \lambda}=\mu j^{\nu}
$$

From this, the current conservation equation can be derived, that is directly connected with the gauge invariance of the system section II f) ii. of main text).

(c) Variation with respect to $x^{\nu}$

To find the equations of fluid motion, we assume the field potential $a_{\nu}$ given and vary only the trajectory of the fluid particle $\left(\boldsymbol{X}\right.$ or $\left.\boldsymbol{v}=\partial_{t} \boldsymbol{X}\right)$. In this case, the third Lagrangian $\mathcal{L}^{(\mathrm{F})}$ of (B.31) is kept unchanged because it depends only on the field $a_{\nu}$. Therefore, the variation under consideration is equivalent to what is done in Appendix B.3. The equation (B.29) derived there is rewritten here:

$$
\nabla_{\tau}^{(\mathrm{pfa})} v^{k} \equiv \mathrm{D}_{t} v^{k}+\rho^{-1} \partial_{k} p-f_{k l} v^{l}=0 .
$$

Thus we find an extended equation of Euler's equation in the presence of background field $a_{\mu}$ giving rise to new third term.

In Appendix C, we see that the equation (C.10) is a geodesic equation of free motion of perfect fluid of a constant density $\rho_{*}$, expressed as $\widehat{\nabla}_{t} \boldsymbol{u} \equiv \partial_{t} \boldsymbol{u}+(\boldsymbol{u} \cdot \nabla) \boldsymbol{u}+\nabla\left(p / \rho_{*}\right)=0$. An analogous equation (B.17) is given for a perfect fluid in Appendix B.2. Just above, we have obtained another analogous equation (B.36). Now we have introduced a new symbol $\nabla_{\tau}^{(\mathrm{pfa})}$ to denote the covariant $\tau$-derivative of perfect fluid in the presence of background field $a_{\mu}$. In fact, the leading term of (B.26) can be written as

$$
\delta \mathcal{J}^{(\mathrm{PFA})}=-\frac{1}{c \sqrt{1-\beta^{2}}} \Delta m\left(\nabla_{\tau}^{(\mathrm{pfa})} v^{k}\right) \mathrm{d} \tau \delta x^{k}=0 .
$$


for the action principle concerned with the geodesic equation of free motion of a perfect fluid in the presence of the background field $a_{\mu}$.

Appendix C. Free motion of physical systems and curved geodesics Appendix C.1. Two free physical systems described by curved geodesics (a) Free motion of a particle in gravity field by Newtonian mechanics

To begin with, consider free motion of a test particle of a unit mass in the Galilean rectangular space $\left(x^{k}\right)$ with a universal absolute time $t$. The particle in free motion in a gravitational field takes a curved trajectory in general according to Newtonian mechanics. By the equation of motion, the particle motion in the Earth's gravity potential $\Phi_{E}\left(x^{k}\right)$ is described by

$$
\frac{\mathrm{d}}{\mathrm{d} t} v^{k}+\frac{\partial \Phi_{E}}{\partial x^{k}}=0, \quad v^{k} \equiv \frac{\mathrm{d} x_{p}^{k}}{\mathrm{~d} t}, \quad k=1,2,3,
$$

where the particle takes a curved trajectory $x_{p}^{k}(t)$ and $v^{k}$ is the $k$-th component of its velocity. In the modern view to take the space and time linked to form a $4 \mathrm{~d}$-continuum, the curved trajectory of a free particle is described as a geodesic curve in the linked space-time. Let us take an illustrative example according to Utiyama (1987, §2.3), and consider a free-falling elevator in the Earth's gravitational field $\Phi_{E}\left(x^{\nu}\right)$. The free-falling elevator provides a particular inertial system of spacetime, in which free motion of a particle is described by

$$
\mathrm{d}^{2} X^{\mu} / \mathrm{d} \tau^{2}=0
$$

where $X^{\mu}$ is the particle coordinates in the frame $F_{\text {el }}$ fixed to the free-falling elevator. The gravity effect does not appear apparently because the acceleration owing to the gravity acting on both of the elevator and the particle are the same and cancel out in the free-falling frame $F_{e l}$. Thus, the particle takes a straight path $X^{\mu}=a^{\mu} \tau+b^{\mu}$ with respect to $F_{e l}$ with $a^{\mu}$ and $b^{\mu}$ being constants.

According to the section $\S 3$ of Part I, let us observe the same motion from another frame, which is the frame $F_{E}$ fixed to the Earth surface, where the coordinates are given by $x^{\mu}$. Suppose that the relation between the two frames $F_{e l}$ and $F_{E}$ is given by the transformation function $X^{\mu}=X^{\mu}\left(x^{\nu}\right)$. Under this transformation from $X^{\mu}$ to $x^{\nu}$, the equation of free motion $\mathrm{d}^{2} X^{\mu} / \mathrm{d} \tau^{2}=0$ in the free-falling frame $F_{e l}$ (where $\tau$ is the proper time defined by (A.2)) is transformed to that of the frame $F_{E}$ as follows,

$$
\frac{\mathrm{d}}{\mathrm{d} \tau} \frac{\mathrm{d} X^{\mu}}{\mathrm{d} \tau}=\frac{\mathrm{d}}{\mathrm{d} \tau}\left[\frac{\partial X^{\mu}}{\partial x^{\nu}} \frac{\mathrm{d} x^{\nu}}{\mathrm{d} \tau}\right]=A_{\nu}^{\mu}\left[\frac{\mathrm{d}^{2} x^{\nu}}{\mathrm{d} \tau^{2}}+\Gamma_{\alpha \beta}^{\nu} \frac{\mathrm{d} x^{\alpha}}{\mathrm{d} \tau} \frac{\mathrm{d} x^{\beta}}{\mathrm{d} \tau}\right]=0
$$

where $A=\left(A_{\nu}^{\mu}\right)$ is a transformation matrix. Using the inverse $A^{-1}$ of $A_{\nu}^{\mu}$ and multiplying by $\left(A^{-1}\right)_{\mu}^{\sigma} \equiv \partial x^{\sigma} / \partial X^{\mu}$, the above equation becomes

$$
\frac{\mathrm{d}^{2} x^{\sigma}}{\mathrm{d} \tau^{2}}+\Gamma_{\alpha \beta}^{\sigma} \frac{\mathrm{d} x^{\alpha}}{\mathrm{d} \tau} \frac{\mathrm{d} x^{\beta}}{\mathrm{d} \tau}=0, \quad \text { where } \quad \Gamma_{\alpha \beta}^{\sigma}=\frac{\partial x^{\sigma}}{\partial X^{\sigma}} \frac{\partial X^{\sigma}}{\partial x^{\alpha} \partial x^{\beta}}=\Gamma_{\beta \alpha}^{\sigma} .
$$

This states that the particle trajectory is curved in general when $\Gamma_{\alpha \beta}^{\sigma} \neq 0$, where $\Gamma_{\alpha \beta}^{\sigma}$ is playing the role of Christoffel symbol of covariant derivatives of Riemannian geometry.

The 4-velocity $u^{\nu} \equiv \mathrm{d} x^{\nu} / \mathrm{d} \tau$ of the particle is defined by (A.3) as $u^{\nu}=$ $(1, \boldsymbol{v} / c) / \sqrt{1-\beta^{2}}$. In the non-relativistic limit as $\beta \ll 1$ for the particle velocity $|\boldsymbol{v}|$ much less than the light velocity $c$, this yields $u^{\nu}=\mathrm{d} x^{\nu} / \mathrm{d} \tau \rightarrow(1, \boldsymbol{v} / c)=v^{\nu} / c$ in the limit $(\mathrm{d} \tau \rightarrow c \mathrm{~d} t)$. In this case, the equation (C.3) becomes

$$
\frac{\mathrm{d}}{\mathrm{d} t} v^{\sigma}+\Gamma_{\alpha \beta}^{\sigma} v^{\alpha} v^{\beta}=0, \quad \text { in particular } \quad \frac{\mathrm{d}}{\mathrm{d} t} v^{k}+\Gamma_{00}^{k} \cdot 1 \cdot 1=0,
$$


where $v^{\sigma}=\mathrm{d} x^{\sigma} / \mathrm{d} t$, the second equation is given for $\sigma=k=1,2,3,(\alpha, \beta)=(0,0)$, and the factors $\Gamma_{\alpha \beta}^{\sigma}$ other than $\Gamma_{00}^{k}$ are set to zero. Compare this with (C.1). By assuming the following relation of equality: $\Gamma_{00}^{k}=\partial \Phi_{E} / \partial x^{k}$, the second equation of (C.4) becomes equivalent to the equation (C.1). From the context of physics of the gravity theory, this is very important because it implies a relation which equates the geometrical term $\Gamma_{00}^{k}$ (called the Christoffel symbol) to a space derivative of the gravity potential $\partial_{k} \Phi_{E}$.

More precisely in mathematics, it is known section III b) of Kambe (2021a)) that a free particle moving in curved spacetime is governed by the geodesic equation of the form

$$
\frac{\mathrm{d}^{2} x^{\alpha}}{\mathrm{d} \lambda^{2}}+\Gamma_{\beta \gamma}^{\alpha} \frac{\mathrm{d} x^{\beta}}{\mathrm{d} \lambda} \frac{\mathrm{d} x^{\gamma}}{\mathrm{d} \lambda}=0 .
$$

Replacing the affine parameter $\lambda$ with an equivalent proper time $\tau$, the equation (C.5) reduces to (C.3). Since the equations (C.1) and (C.4) have the form of the geodesic equation (C.5), one can read off

$$
\Gamma_{00}^{k}=\partial \Phi_{E} / \partial x^{k} \quad(k=1,2,3) ; \quad \text { all other } \Gamma_{\beta \gamma}^{\alpha} \text { vanish. }
$$

According to section III b)i. of Part I, in a curved 4d spacetime, a covariant derivative of a vector field $v^{\alpha}\left(x^{\mu}\right)$ along a curve $P(\lambda)$ with its tangent $u^{\beta}=\mathrm{d} x^{\beta} / \mathrm{d} \lambda$ is defined by

$$
\left(\widehat{\nabla}_{\boldsymbol{u}} \boldsymbol{v}\right)^{\alpha} \equiv \frac{\mathrm{d}}{\mathrm{d} \lambda} v^{\alpha}+\Gamma_{\beta \gamma}^{\alpha} v^{\beta} u^{\gamma} \equiv \widehat{\nabla}_{\lambda}
$$

where $\widehat{\nabla}$ denotes the nabla-operator in the $4 \mathrm{~d}$ spacetime. Using this definition, the geodesic equation (C.5) can be written simply as

$$
\widehat{\nabla}_{\boldsymbol{u}} \boldsymbol{u}=0, \quad \text { or } \quad \widehat{\nabla}_{\lambda} \boldsymbol{u}=0, \quad \text { where } \quad u^{\alpha} \equiv \mathrm{d} x^{\alpha}(P) / \mathrm{d} \lambda .
$$

According to the differential geometry (Misner et al. 2017, Chap.8), this states that the geodesic is a curve $P(\lambda)$ which parallel-transports its tangent $u^{\alpha}=\mathrm{d} x^{\alpha}(P) / \mathrm{d} \lambda$. In the flat space of special relativity where $g_{\mu \nu}$ is given by the metric $\eta_{\mu \nu}=\operatorname{diag}(-1,1,1,1)$, the geodesic takes a straight path $\mathrm{d}^{2} x^{\alpha} / \mathrm{d} \lambda^{2}=0$, since $\Gamma_{\beta \gamma}^{\alpha}=0$ (see the footnote $\dagger$ ).

The equation (C.5) can be written in the form of a geodesic equation, by taking the specification of $\lambda=t, u^{\alpha}=\left(1, v^{k}\right)$ with $v^{k}=\mathrm{d} x^{k}(P) / \mathrm{d} t$ and the equation (C.6):

$$
\left(\widehat{\nabla}_{\boldsymbol{u}} \boldsymbol{u}\right)^{k} \equiv \frac{\mathrm{d}}{\mathrm{d} t} u^{k}+\Gamma_{00}^{k} u^{0} u^{0}=0, \quad u^{0}=1, \quad u^{k} \equiv \frac{\mathrm{d} x^{k}(P)}{\mathrm{d} t}=v^{k}
$$

where the operator $\widehat{\nabla}$ is defined by (C.7). This implies that the free motion of a test particle in a gravitational potential field $\Phi_{E}\left(x^{k}\right)$ is described by a geodesic trajectory which is curved in a linked space-time. A geodesic curve is a generalization of a straight line in flat spacetime to a curved spacetime.

With a simplified potential $\Phi=g z$ of a uniform value $g$ of gravity in a cartesian $(x, y, z)$-space, one finds a trajectory of a point-mass: $z(t)=\frac{1}{2} g t^{2}+w_{0} t, x(t)=u_{0} t$ and $y(t)=v_{0} t$ where $\left(u_{0}, v_{0}, w_{0}\right)$ denote the initial velocity. Thus it is seen that the parabolic trajectory of free motion of a point-mass in the gravity field $\Phi=g z$ is a geodesic in the linked spacetime $(t, x, y, z)$, which is curved in a geometrical sense.

\section{(b) Free motion of a perfect fluid in a flat space}

Let us consider free motion of a perfect fluid of constant density under pressure field in a flat space. This case is worth given a particular remark, because this is a free motion characterized with curvature tensors that occurs in a flat 3-space for a perfect

$\dagger$ The equation (3.12) of Part I paper: $\Gamma_{\beta \gamma}^{\alpha}=g^{\alpha \mu} \Gamma_{\mu \beta \gamma}, \quad \Gamma_{\mu \beta \gamma}=\frac{1}{2}\left(\frac{\partial g_{\mu \beta}}{\partial x^{\gamma}}+\frac{\partial g_{\mu \gamma}}{\partial x^{\beta}}-\frac{\partial g_{\beta \gamma}}{\partial x^{\mu}}\right)$. 
fluid under pressure field. This is formulated mathematically with an exact analysis by Arnold (1966).

In $\S 2.1$, we have seen that free motion of a perfect fluid can be described by the Euler's equation of motion of the form (2.2), which is analogous to the Newton's equation of motion (C.4) rewritten in the geometrical language, transformed from the original Newton equation (C.1).

This implies that the Euler's equation of motion too may be further transformed to the form of geodesic equation (C.9), because both equations are descriptions of free motion. This is true. In fact, it is already down by the mathematician V.I. Arnold (1966) for a perfect fluid of constant density satisfying the condition of incompressibility by applying the differential geometry of Lie groups of infinite dimensions.

According to Arnold, it is found on the basis of Riemannian geometry and Lie group theory that the Euler's equation of motion for flows of a perfect fluid of uniform density on a bounded flat space-time $M$ is a geodesic equation on a group of volume-preserving diffeomorphisms with the metric of the kinetic energy (see also Kambe (2010, Chap. 8) for some details, in addition to Arnold (1966) ). Here, we consider the fluid motion in flat space.

Defining $\boldsymbol{u}(\boldsymbol{x})$ as the 3-velocity field for $\boldsymbol{x} \in M$ (a bounded 3-space) satisfying the divergence-free condition $\operatorname{div}_{x} \boldsymbol{u}(\boldsymbol{x})=0$ for a constant density $\rho_{*}$, the geodesic equation is given by

$$
\widehat{\nabla}_{t} \boldsymbol{u} \equiv \partial_{t} \boldsymbol{u}+\bar{\nabla}_{\boldsymbol{u}} \boldsymbol{u}=0, \quad \bar{\nabla}_{\boldsymbol{u}} \boldsymbol{u} \equiv(\boldsymbol{u} \cdot \nabla) \boldsymbol{u}+\nabla\left(p / \rho_{*}\right),
$$

(eq. (8.42) of Kambe (2010)), where $\bar{\nabla}$ is a divergence-free connection satisfying

$$
\operatorname{div}\left(\bar{\nabla}_{\boldsymbol{u}} \boldsymbol{u}\right)=\operatorname{div}\left((\boldsymbol{u} \cdot \nabla) \boldsymbol{u}+\nabla\left(p / \rho_{*}\right)\right)=0 .
$$

This ensures $\operatorname{div} \boldsymbol{u}=0$ at any time from (C.10) if it is satisfied initially. The equation (C.11) constrains the pressure field $p$ to satisfy $\nabla^{2} p=-\rho_{*} \partial_{j} \partial_{k}\left(u^{j} u^{k}\right)$. The geodesic equation (C.10) is nothing but the Euler's equation of a perfect fluid of constant density:

$$
\partial_{t} \boldsymbol{u}+(\boldsymbol{u} \cdot \nabla) \boldsymbol{u}+\nabla\left(p / \rho_{*}\right)=0 .
$$

Using the operator $\mathrm{D}_{t}$ of (2.4), this can be rewritten in the form of equation-of-motion of a fluid particle analogous to $(2.2)$ as $\mathrm{D}_{t} \boldsymbol{u}+\nabla P=0$, where $P \equiv p / \rho_{*}$. Since this is analogous to (C.1), it is suggested that the geodesic equation (C.12) can be rewritten in the form of an equation using a covariant derivative $\widehat{\nabla}$ of 4 -spacetime in terms of Christoffel symbol $\Gamma$ 's.

For that purpose, we define the 4-velocity by $v^{\nu}=\mathrm{d} X^{\nu} / \mathrm{d} t=(c, \boldsymbol{u})$, in the nonrelativistic limit $(\beta \rightarrow 0)$ with $\mathrm{d} X^{\nu}=(c \mathrm{~d} t, \mathrm{~d} \boldsymbol{X})$. Then, the equation (C.12) can be rewritten in the following form of geodesic equation:

$$
\begin{aligned}
\left(\widehat{\nabla}_{v} v\right)^{k} & \equiv \frac{\mathrm{D}}{\mathrm{D} t} v^{k}+\Gamma_{00}^{k} v^{0} v^{0}=0, \quad \text { for } \quad k=1,2,3 \\
v^{0} & =c, \quad \Gamma_{00}^{k}=c^{-2}\left(\partial / \partial x^{k}\right) P, \quad \text { all other } \Gamma_{\beta \gamma}^{\alpha} \text { vanish. }
\end{aligned}
$$

Appendix C.2. Free dynamics and action principle of invariant variations

Free motion of a perfect fluid under a pressure field $p\left(x^{\nu}\right)$ and a background field $a_{\mu}\left(x^{\nu}\right)$ in a flat space was studied in section II c) and f), where modified Euler's equation of motion (2.11) was derived in the former section II c) and the equation (2.32) governing the background field was derived in the latter. In particular, the set of latter equations re-ensures the continuity equation. The same continuity equation was required already in section II a) from the invariance of the interaction action $S^{(\mathrm{int})}$ to the gauge transformation of $a_{\mu}\left(x^{\nu}\right)$. 
By the scenario of Utiyama's gauge principle, the new field $a_{\mu}$ should be incorporated as a connection term in a covariant derivative. To that end, physical and mathematical formulation are presented by geometrical language in this section enabling the generalization. As a matter of fact, this section aims to conclude the fluid gauge theory.

The Euler's equation (2.2) can be written as $\mathrm{D}_{t} \boldsymbol{v}+\rho^{-1} \nabla p=0$ in the form of an equation of motion of a fluid particle and describes free motion of a perfect fluid. In principle, free motion is given by a geodesic equation. Appendix B.2 derives the same equation (B.17) as a geodesic equation deduced from the action principle. In mechanical systems, the variational principle of action integrals yields a geodesic equation.

Naively speaking, a geodesic is a curve representing the shortest (or extremum) path between two points in a Riemannian manifold. More generally, a geodesic is defined to be a curve whose tangent vector $T$ remain parallel if they are transported along it, i.e. if $\widehat{\nabla}_{T} T=0$ (see Kambe (2021a): Eq.(4.8) and Appendix A.6). This recovers the statement mentioned above that the arc length between two points in a Riemannian manifold takes the extremum length when $\widehat{\nabla}_{T} T=0$. This fact is seen transparently in the definition of action integral of a point mass of Eq.(B.1) of Appendix B.1:

$$
S^{(\mathrm{m})}=-c m \int \mathrm{d} \tau, \quad \mathrm{d} \tau \equiv \sqrt{-\mathrm{d} x_{\nu} \mathrm{d} x^{\nu}}=c \mathrm{~d} t \sqrt{1-\beta^{2}}, \quad \beta \equiv \frac{v}{c},
$$

where $\mathrm{d} \tau$ is the relativistic infinitesimal time-like interval, invariant by Lorentz transformation. Vanishing variation of the integral, $\delta \int_{a}^{b} \mathrm{~d} \tau=0$, signifies the extremum of the time-like interval between the end points $a$ and $b$. Hence there is no doubt that a geodesic equation such as $\widehat{\nabla}_{T} T=0$ plays a role in the variation.

\section{(a) Free particle of mass $m$}

In the Appendix B, it is found that the variation of $S^{(\mathrm{m})}$ is given by (B.5):

$$
\delta S^{(\mathrm{m})}=-c m \int_{a}^{b} \frac{\mathrm{d} u^{\nu}}{\mathrm{d} \tau} \delta x^{\nu} \mathrm{d} \tau,
$$

under the condition that the variation $\delta x^{\nu}$ vanishes at end points. Requiring $\delta S^{(\mathrm{m})}=0$ for arbitrary variation $\delta x^{\nu}$, we obtain the geodesic equation:

$$
\frac{\mathrm{d} u^{\nu}}{\mathrm{d} \tau}=\nabla_{\tau} u^{\nu}=u^{\nu} \partial_{\nu} u^{\nu}=0, \quad \frac{\mathrm{d}}{\mathrm{d} \tau}=\nabla_{\tau}=u^{\nu} \partial_{\nu},
$$

Note that a tangent vector $T$ is defined as $T=T^{\nu} \partial_{\nu}=u^{\nu} \partial_{\nu}=\left(\mathrm{d} x^{\nu} / \mathrm{d} \tau\right)\left(\partial / \partial x^{\nu}\right)=\mathrm{d} / \mathrm{d} \tau$, and that the above equation can be written also in the form, $\widehat{\nabla}_{T} T=0$ with $\widehat{\nabla}_{T}=T^{\nu} \partial_{\nu}$. Thus, it is found that a free particle is governed by the geodesic equation: $\nabla_{\tau} u^{\nu}=0$.

\section{(b) Free motion of a perfect fluid}

The action of a perfect fluid is given by (B.7) and (B.8) as

$$
S^{(\text {pf })}=-c \iint(\rho \mathrm{d} \mathcal{V})\left(1+c^{-2} \bar{\epsilon}(\bar{\rho})\right) \mathrm{d} \tau, \quad \rho \mathrm{d} \mathcal{V}=\mathrm{d} m
$$

Comparing this action for a perfect fluid (of continuum material of density $\rho$ ) with the action $S^{(\mathrm{m})}$ of (C.14) for a single particle of mass $m$, one finds that the mass energy $m c^{2}$ is replaced by an integral of the energy $\rho\left(c^{2}+\bar{\epsilon}\right)$ per a volume element $\mathrm{d} \mathcal{V}$, where $\bar{\epsilon}$ is the specific internal energy in the rest frame of the fluid. Namely, the internal energy is added to the mass energy because the fluid has its own thermal energy in addition to the rest-mass energy. This is the difference of the two systems.

In the Appendix B.2, the integrand of variation $\delta S^{(\mathrm{pf})}$ is given by (B.14). Deleting vanishing terms, its dominant leading order term is given by

$$
\delta \mathcal{J}^{(\mathrm{pf})}=-c(\Delta m)\left(\frac{\mathrm{d}}{\mathrm{d} \tau} u_{\nu}+c^{-2} \frac{1}{\rho} \partial_{\nu} p\right) \delta x^{\nu} \mathrm{d} \tau, \quad \Delta m=\rho \Delta \mathcal{V} .
$$


For the invariance of the action $S^{(\mathrm{pf})}$, it is required that $\delta \mathcal{J}^{(\mathrm{pf})}=0$ is satisfied for arbitrary variation $\delta x^{\nu}$. Thus, we obtain the following geodesic equation:

$$
\nabla_{\tau}^{(\mathrm{pf})} u^{\nu} \equiv \frac{\mathrm{d}}{\mathrm{d} \tau} u^{\nu}+c^{-2} \frac{1}{\rho} \partial_{\nu} p=0, \quad \frac{\mathrm{d}}{\mathrm{d} \tau}=u^{\nu} \partial_{\nu}=\frac{1}{c}\left(\left(\partial_{t}+v^{k} \partial_{k}\right)+O\left(\beta^{2}\right)\right) .
$$

This reduces to the Euler's equation of motion of (2.2), which is the same as Eq. (B.17) deduced in Appendix B.2. As the above derivation shows clearly, the equation (C.17) defines the covariant derivative $\nabla_{\tau}^{(\mathrm{pf})} u^{\nu}$ of the perfect fluid. The derivative may be called more appropriately as an invariant derivative (Utiyama (1987) Chap.11), because the invariance of the action $S^{(\mathrm{pf})}$ is ensured by (C.17).

(c) Free motion of perfect fluid under a background field $a_{\nu}$

The action of this system is given in section II f) by (2.28):

$$
S^{(\text {total })}=S^{(\mathrm{pf})}+S^{(\mathrm{int})}+S^{(\mathrm{F})}=\int\left[\int\left(\mathcal{L}^{(\mathrm{pf})}+\mathcal{L}^{(\mathrm{int})}+\mathcal{L}^{(\mathrm{F})}\right) \mathrm{d} \mathcal{V}\right] c \mathrm{~d} t,(\mathrm{C} .18)
$$

where $\mathcal{L}^{(\mathrm{pf})}, \mathcal{L}^{(\mathrm{int})}$ and $\mathcal{L}^{(\mathrm{F})}$ are defined by $(2.27)$.

(i) To find the equations governing the background field $a_{\nu}$, we take variation of the total action $S^{(\text {total })}$ by assuming the fluid motion given and vary the potential $a_{\nu}$ only. Expressing the integrand of variation of $S^{(\mathrm{int})}$ and $S^{(\mathrm{F})}$ by $\mathcal{J}^{(\mathrm{int})}$ and $\mathcal{J}^{(\mathrm{F})}$ respectively, and using (2.31) in $\S 2.6$, their variations are given by

$$
\delta \mathcal{J}^{(\mathrm{int})}+\delta \mathcal{J}^{(\mathrm{F})}=\left(j^{\nu}-\frac{1}{\mu} \frac{\partial}{\partial x^{\lambda}} f^{\nu \lambda}\right) \delta a_{\nu} \mathrm{d} \tau c^{-1} \mathrm{~d} \overline{\mathcal{V}}, \quad \mathrm{d} \mathcal{V}=\mathrm{d} \overline{\mathcal{V}} \sqrt{1-\beta^{2}}
$$

Vanishing of $\delta \mathcal{J}^{(\mathrm{int})}+\delta \mathcal{J}^{(\mathrm{F})}=0$ for arbitrary variation $\delta a_{\nu}$ is given in $\S 2.6$ as

$$
\frac{\partial}{\partial x^{\lambda}} f^{\nu \lambda}=\mu j^{\nu}
$$

(see (2.29) for the 4-current $j^{\nu}$ ). This includes two important messages inside.

Firstly, the equation yields the law of current conservation $\partial_{\nu} j^{\nu}=0$ of $(2.33)$, which is rewritten in the following equation of continuity

$$
\partial_{\nu} j^{\nu}=0 \quad \Rightarrow \quad \partial_{t} \rho+\nabla \cdot \boldsymbol{j}=0,
$$

for $j^{\nu}=(\rho c, \rho \boldsymbol{v})$ with $\boldsymbol{j}=\rho \boldsymbol{v}$. Secondly, the equation (C.20) represents a pair of fluid Maxwell equations. Using the definition (3.14) of the fields $\boldsymbol{e}$ and $\boldsymbol{b}$ and the matrix representation (3.15) of $f^{\nu \lambda}$, the equation (C.20) represents the followings:

$$
\varepsilon \nabla \cdot \boldsymbol{e}=\rho, \quad-\varepsilon \partial_{t} \boldsymbol{e}+\mu^{-1} \nabla \times \boldsymbol{b}=\boldsymbol{j}, \quad \text { where } \varepsilon=1 /\left(c^{2} \mu\right) .
$$

(ii) Next, to find the equations of fluid motion, we assume the field potential $a_{\nu}$ given and vary only the trajectory of the fluid particle $\left(\boldsymbol{X}\right.$ or $\left.\boldsymbol{v}=\partial_{t} \boldsymbol{X}\right)$. This is done in Appendix B.3 or in section III c) with the integrand variation $\delta \mathcal{J}^{(\mathrm{fl}+\mathrm{a})}$ given by $(2.10)$ or by (B.26). For the invariance of the action, vanishing of $\delta \mathcal{J}^{(\mathrm{fl}+\mathrm{a})}=0$ is required for arbitrary variation $\delta x^{\nu}$. Thus, we obtain the following geodesic equation:

$$
\nabla_{\tau}^{(\mathrm{fl}+\mathrm{a})} u^{\nu} \equiv \frac{\mathrm{d}}{\mathrm{d} \tau} u_{\nu}+c^{-2} \frac{1}{\bar{\rho}} \partial_{\nu} \bar{p}-c^{-1} f_{\nu \mu} u^{\mu}=0 .
$$

For the definition of $\mathrm{d} / \mathrm{d} \tau$, see (C.17). In section III c), it is already shown that this reduces to the modified Euler equation (3.16) with additional term of fluid Lorentz force $\boldsymbol{f}_{a}$ :

$$
\mathrm{D}_{t} \boldsymbol{v}+\rho^{-1} \nabla p-\boldsymbol{f}_{a}=0, \quad \quad \boldsymbol{f}_{a} \equiv \boldsymbol{e}+\boldsymbol{v} \times \boldsymbol{b} .
$$




\section{References Références Referencias}

1. Aitchison IJ R and Hey A J G 2013 Gauge Theories in Particle Physics: A Practical Introduction, Vol. 1 \& 2, Fourth Edition. (CRC Press, Taylor \& Francis Group).

2. Arnold V I 1966 Sur la gèomètrie diffèrentielle des groupes de Lie de dimension infinie et ses applications a l'hydrodynamique des fluides parfaits. Ann. Inst. Fourier (Grenoble) 16, 319-361.

3. Del jAlamo, J. C. and Jimjenez, J., Linear energy amplification in turbulent channels. J. Fluid Mech. 2006, 559, 205-213.

4. Hutchins, N. and Marusic, I., Evidence of very long meandering features in the logarithmic region of turbulent boundary layers. J. Fluid Mech. 2007, 579, 1-28.

5. Jackson J D 1999 Classical Electrodynamics (Third edition) (John Wiley \& Sons).

6. Kambe T 2010 Geometrical Theory of Dynamical Systems and Fluid Flows, (World Scientific).

7. Kambe T 2011 A new solution of Euler's equation of motion with explicit expression of helicity, WSEAS Special ISSue "Mathematical Fluid Dynamics 53716; or WSEAS Transactions on Fluid Mechanics 7(2), 59-70.

8. Kambe T 2013 New representation of rotational flow fields satisfying Euler's equation of an ideal compressible fluid, Fluid Dyn. Res. 45, 015505 (16pp).

9. Kambe T 2017 New scenario of turbulence theory and wall-bounded turbulence: Theoretical significance, Geophys. Astrophys. Fluid Dyn. 111, 448-507.

10. Kambe T 2020 New perspectives on mass conservation law and waves in fluid mechanics, Fluid Dyn. Res. 52, 1 - 34 (34pp).

11. Kambe T 2021a: Gauge symmetries in physical fields (review), Global Journal of Science Frontier Research, vol.21, iss.4.

12. Kambe T 2021b: "Fluid Gauge Theory (updating title): New Perspective on Mass Conservation and Rotational Waves", presented at ICTAM Milano 2020+1 virtual, Aug. 2021: DOI: 10.13140/RG.2.2.28629.35047. In "Tsutomu Kambe The University of Tokyo - Todai -ResearchGate"

13. Kim, K.C. and Adrian, R.J., Very large-scale motion in the outer layer. Phys. Fluids 1999, 11, 417-422.

14. Kundt, A. 1866: Ueber eine neue Art Akustischer Staubfiguren und uber die Anwendung derselben zur Bestimmung der Shallgeschwindigkeit in festen Korpern und Gasen, Annalen der Physik und Chemie, vol.127 (4): $497 ? 523$. doi:10.1002/andp.18662030402.

15. Landau L D and Lifshitz E M 1975 The Classical Theory of Fields (Pergamon Press).

16. Landau L D and Lifshitz E M 1987 Fluid Mechanics (Pergamon Press, 2nd ed.).

17. Misner C W, Thorne KS and Wheeler J A 2017 Gravitation (W.H. Freeman and Co., San Francisco).

18. Monty, J.P., Stewart, J.A., Williams, R.C. and Chong, M.S., Large-scale features in turbulent pipe and channel flow. J. Fluid Mech. 2007, 589, 147-156.

19. Noether E 1918 Invariant variations problem, Nachr. d. König. Gesellsch. d. Wiss. zu Göttingen, Math-phys. Klasse, 235-257.

20. Reynolds, W.C. and Hussain, A.K.M.F., The mechanics of an organized wave in turbulent shear flow. Part 3. Theoretical models and comparisons with experiments. J. Fluid Mech. 1972, 54, 263 - 288.

21. Scofield, D.F. and Huq, P., Fluid dynamical Lorentz force law and Poynting theorem ?derivation and implications. Fluid Dyn. Res. 2014, 46, 055514 (22 $\mathrm{pp})$. 
22. Smits, A. J., McKeon B. J. \& Marusic, I., High-Reynolds number wall turbulence. Annu. Rev. Fluid Mech. 2011, 43, 353-375.

23. Utiyama R 1956 Invariant theoretical interpretation of interaction, Phys. Rev. $101,1597-1607$.

24. Utiyama R 1987 Ippan Gauge ba ron josetsu (Introduction to the General Gauge Field Theory) (Iwanami Shoten, Tokyo). 